\title{
Characterization of the MAPK Gene Family and PbrMAPK13 Response to Hormone and Temperature Stresses via Different Expression Pattern in Pyrus $\times$ bretschneideri Pollen
}

\author{
Jin Jiao, Xing Liu, and Juyou Wu \\ College of Horticulture, State Key Laboratory of Crop Genetics and Germplasm Enhancement, \\ Nanjing Agricultural University, Nanjing 210095, China \\ Guohua $\mathrm{Xu}^{1}$ \\ Jiangsu Key Laboratory of BioFunctional Molecule, Jiangsu Second Normal University, Nanjing \\ 210013, China \\ Shaoling Zhang ${ }^{1}$ \\ College of Horticulture, State Key Laboratory of Crop Genetics and Germplasm Enhancement, \\ Nanjing Agricultural University, Nanjing 210095, China
}

\begin{abstract}
Additional Index words. antisense, bioinformatics, chinese white pear, genomic evolution, phytohormones, Rosaceae
Abstract. Mitogen-activated protein kinase (MAPK) cascades are universal signal-transduction modules, but the available information is limited in pear (Pyrus). In this study, 87 MAPK genes were identified from five Rosaceae species: chinese white pear (Pyrus $\times$ bretschneideri cv. Dangshansuli), peach (Prunus persica), apple (Malus domestica), strawberry (Fragaria vesca), and plum (Prunus mume), 23 of which came from chinese white pear, designated as PbrMAPK. Based on the phylogenetic analysis and the architectures of conserved protein motifs of these gene sequences, MAPK family genes of five Rosaceae species were classified into two primary types (I and II) or four groups (Classes A-D). We have indicated that both segment and tandem duplications significantly contributed to the expansion of the MAPK family in Rosaceae by analysis of genomic evolution. In chinese white pear pollen, the expression analysis revealed that all PbrMAPKs could respond to temperature stresses (high/low temperature) and phytohormones, except PbrMAPK8 and PbrMAPK19 that displayed lower expressions, which suggested that PbrMAPKs play pivotal roles in signal-transduction pathways. In addition, we determined that PbrMAPK13 is located in the nucleus and plasma membranes. The lengths of pollen tubes became shorter when PbrMAPK13 was silenced by antisense oligonucleotide transfection. Our results provided an evolutionary foundation and functional characterization for MAPK gene families in chinese white pear and other plant species so as to elucidate their biological roles.
\end{abstract}

Mitogen-activated protein kinase cascades play key roles in regulating various biotic and abiotic stresses during the plant life cycle. These cascades are highly conserved signaling components in all eukaryotic cell signal transduction. They can be linked to cell responses and different cell-membrane receptors and take a leading role in endogenous and exogenous signaling (Zhang et al., 2006). Three classes of functionally related kinases MAPKs, MAPK kinases, MAPKKs, and MAPKK kinases form signal-transmission cascades to regulate efficiently amplifying, integrating, and channeling information during the process of cell development, metabolism, and response (Robinson and Cobb, 1997). As dual-specificity kinases, MAPKKs activate an MAPK in the activation loop by the double phosphorylation of the T-X-Y motif (Colcombet and Hirt, 2008).

MAPK cascades are universal signal-transduction modules in eukaryotes. Plant cells respond to environmental stresses

Received for publication 20 Oct. 2016. Accepted for publication 21 Feb. 2017. The work was financially supported by the National High-Tech R\&D Program of China (863 Program, 2013AA102606-02), China, and Agriculture Research System (CARS-29).

${ }^{1}$ Corresponding authors. E-mail: slzhang@njau.edu.cn; njxgh@jssnu.edu.cn. (such as wounding, drought, and temperature) and growth signals [such as auxin, ethylene, and abscisic acid (ABA)] via the receptor protein kinase; thus, the MAPK cascade is rapidly activated. To date, the responses of many MAPK genes to abiotic stress and their molecular signaling pathways have been widely studied in several model plants. AtMAPK3/4/6 in arabidopsis (Arabidopsis thaliana) was the most extensively studied and included their orthologs in other plant species. For example, the MAPKK4/MAPKK5-MAPK3/MAPK6 cascade is also involved in the regulation of 1-aminoacyclopropane-1carboxylate synthase activity to induce ethylene biosynthesis under pathogen attack (Hord et al., 2008). The YODAMAPKK4/MAPKK5-MAPK3/MAPK6 cascade promoted organ shape and regulated inflorescence architecture as well as localized cell proliferation through acts downstream of the estrogen receptor (Meng et al., 2012). In rice (Oryza sativa), OsMAPK6 was found to be involved in the development of rice, particularly in plant height and grain size (Liu et al., 2015).

Because of the vital regulatory functions of MAPK genes in plant responses to different developmental processes and stresses, in the model plant arabidopsis (20 MAPK), the MAPK gene family has been extensively studied (Ichimura, 2002), as well as in nonmodel plants such as rice (16 MAPK) (Liu and 
Xue, 2007), apple (26 MAPK), and mulberry [Morus notabilis (10 MAPK)] (Wei et al., 2014; Zhang et al., 2013). In comparison with that in other species, the MAPK gene family in Rosaceae has not been widely examined, except in the apple. In the member of the Rosaceae family, pear, it is also the thirdmost economically important fruit in temperate areas. Lately, the draft genome sequence of chinese white pear has been decoded, providing an excellent opportunity for analyzing the MAPK genes in Rosaceae species (Wu et al., 2013).

In the present study, 23 MAPK members were discovered, all sequenced from chinese white pear. Based on the phosphorylation motif and phylogenetic analysis of amino acid sequence, we divided MAPKs into four subfamilies (A-D). Furthermore, comprehensive analysis was performed to characterize Rosaceae MAPK genes and protein sequence features, multiple alignment, domain, phylogenetic relationships, and their expansion and evolutionary history.

To verify their functions in pollen, we analyzed the expression pattern in pollens of chinese white pear MAPK genes by quantitative polymerase chain reaction (qPCR) in response to temperature stresses and various hormonal changes. In addition, specific gene knockdown mediated by the antisense oligodeoxynucleotides (AS-ODN) strategy recently emerged as a rapid and effective tool for probing the gene function in plant cells, particularly for tip-growing pollen tubes. Subsequently, the function of individual genes was studied using this technology.

Based on the genome-wide analysis, we studied the chinese white pear MAPK family, which provided valuable information for understanding the classification and putative function of PbrMAPK.

\section{Materials and Methods}

IDENTIFICATION OF MAPK GENES IN THE ROSACEAE FAMILY. To identify members of the MAPK gene family, multiple database searches were conducted. The arabidopsis MAPK (AtMAPKs) gene sequences acquired from The Arabidopsis Information Resource (TAIR) database (Garcia-Hernandez et al., 2002) were used as queries to perform repetitive basic local alignment search tool (BLAST) searches against the chinese white pear genome dataset (Wang et al., 2007). Protein BLAST (BLASTP) and translated nucleotide BLAST (TBLASTN) were used to search against genome databases with the e-value cutoff set to $1 \mathrm{e}^{-003}$ (Altschul et al., 1990). All retrieved nonredundant sequences were acquired from the Phytozome version 9.1 database (Qiao et al., 2015) and subjected to analyze conserved domains with two different domain databases: the database of protein families (Pfam) 27.0 (Bateman et al., 2004) and simple modular architecture research tool (SMART), with the default parameters (Danquah et al., 2014). Some genes were rejected if they have a size below $<200$ amino acid domains or without MAPK-specific S_TKc domains. The amino acid sequences of the MAPKs from arabidopsis, chinese white pear, apple, peach, strawberry, and plum were downloaded from Phytozome (Qiao et al., 2015).

Conserved DOMaIn AND PHYLOGENETIC ANALYSIS. We used the GeneDoc program, a full-featured multiple-sequence alignment editor, to investigate the amino acid sequences and kinase domains of PbrMAPKs (Nicholas et al., 1996). The MAPK family protein sequence alignments and the phylogenetic tree were created using the multiple sequence comparison by log-expectation
(MUSCLE) program (Edgar, 2004). The phylogenetic trees for MAPKs from arabidopsis, chinese white pear, peach, apple, strawberry, and plum were constructed using the maximum likelihood (ML) method and assessed by bootstrap analysis with 1000 resampling replicates.

Chromosomal location and SYNTENy ANALYSIS. The chromosomal locations of the MAPK genes obtained from genome annotations were displayed using Circos (Qiao et al., 2015). The analysis of synteny among the five Rosaceae genomes was conducted locally using a method similar to that developed for the plant genome duplication database (Lee et al., 2013). First, BLASTP was performed against self and nonself proteomes to search for potential homologous gene pairs $\left(E<1 \mathrm{e}^{-5}\right.$, top five matches) across self and nonself genomes. These homologous pairs were then used for the multiple collinearity scan toolkit (MCScanX) to identify syntenic chains (Wang et al., 2012a). MCScanX was further used to identify whole-genome, segmental, tandem, proximal, and dispersed duplications in the MAPK gene family.

Calculating Ka and Ks of the MAPK gene family. The Ka value (nonsynonymous substitutions per site)/Ks value (synonymous substitutions per site) calculator was applied to estimate the selection pressure of MAPK gene pairs. The algorithm used was the nonuniform grid (Wang et al., 2012b).

Plant materials and stress treatments. All materials were sourced from the chinese white pear trees (cv. Dangshansuli) grown at the Jiangpu base of Nanjing Agricultural University, Nanjing, China. The harvested seeds germinated for $2 \mathrm{~d}$ were transferred to pots containing soil and vermiculite. Roots, leaves, and stems were harvested at $50 \mathrm{~d}$ after transferring. The mature seeds were harvested $\approx 12$ weeks after flowering. Flowers were collected a few days before anthesis, and the styles were detached, weighed, and stored in liquid nitrogen. Anthers were collected, dehisced, and dried in bottles containing desiccants and stored at $-20{ }^{\circ} \mathrm{C}$ over silica gel until needed. Mature pollen was incubated in liquid culture for germination and growth. The culture contained the following components (millimolar): $0.55 \mathrm{Ca}\left(\mathrm{NO}_{3}\right)_{2}, 1.60 \mathrm{H}_{3} \mathrm{BO}_{3}, 1.60$ $\mathrm{MgSO}_{4}, 1.00 \mathrm{KNO}_{3}$ (Shoude, Nanjing, China), 440 sucrose, and 52-(N-morpholino)ethanesulfonic acid hydrate (MES) at pH 5.8 (adjusted with Tris). The pollen was incubated in small petri dishes at $24 \pm 1{ }^{\circ} \mathrm{C}$ at different times (Fan et al., 2001; Lopez-Bucio et al., 2014). The samples were then centrifuged, the supernatant was removed, and the pollen-tube precipitate was frozen in liquid nitrogen for later use. All harvested materials were used for the extraction of total RNA. For quantitative reverse-transcription PCR (RT-PCR) analysis, total RNA was extracted from pollen using RNAqueous (Ambion, Shanghai, China), and other tissues using a TRIzol reagent (Invitrogen, Carlsbad, CA) according to the manufacturer's instructions. Pollen was hydrated and grown as previously described in germination medium (GM) for $1 \mathrm{~h}$ before challenged with temperature or phytohormones. For hormonal treatment, pollen was sprayed with five different hormones including brassinosteroid [BR $\left.\left(0.05 \mu \mathrm{mol} \cdot \mathrm{L}^{-1}\right)\right]$, ABA $(0.2$ $\left.\mathrm{mmol} \cdot \mathrm{L}^{-1}\right)$, indole-3-acetic acid [IAA $\left(0.08 \mathrm{mmol} \cdot \mathrm{L}^{-1}\right)$ ], spermine [SPM $\left(0.005 \mathrm{mmol} \cdot \mathrm{L}^{-1}\right)$ ], spermidine [SPD $(0.05$ $\left.\left.\mathrm{mmol} \cdot \mathrm{L}^{-1}\right)\right]$, and an equivalent concentration of dimethyl sulfoxide as a control (Sigma-Genosys, Beijing, China). For temperature stress treatment, hydrated pollen was cultivated at 17 and $37{ }^{\circ} \mathrm{C}$ on a horizontal shaker ( $\left.\approx 200 \mathrm{rpm}\right)(\mathrm{ZQZY}-70 \mathrm{BF}$; Zhichu, Nanjing, China) for $4 \mathrm{~h}$. This temperature range met the 
scope of our study since chinese white pear tree blossoms generally in April by nature. The samples were placed in liquid nitrogen immediately after sampling and stored at $-80{ }^{\circ} \mathrm{C}$ for total RNA isolation. For the pollen growth assay, the lengths of more than 100 pollen tubes per sample were measured for $4 \mathrm{~h}$ after germination using ImageJ software (Olympus, Shenzhen, China) (Abramoff et al., 2004; Kosetsu et al., 2010).

Quantitative Real-time PCR (QPCR) anAlysis of MAPK GENE EXPRESSION. Samples grounded in liquid nitrogen were used in a mortar and pestle. Total RNA (4 mg) isolated using TRIzol reagent (Invitrogen) was treated with RNase-free DNase I (TaKaRa, Suzhou, China) for $15 \mathrm{~min}$ to eliminate contaminating DNA. The M-MLV reverse transcriptase (Promega, Madison, WI) was used to transcribe the RNA to cDNA reversely, which was used for gene-expression analysis by qPCR. qPCR system (Applied Biosystems, Foster City, CA) and fluorescent dye SYBR Green (Toyobo, Osaka, Japan) were applied in our qPCRs with the following program: $95{ }^{\circ} \mathrm{C}$ for $30 \mathrm{~s}, 95^{\circ} \mathrm{C}$ for $5 \mathrm{~s}$, and $60^{\circ} \mathrm{C}$ for $45 \mathrm{~s}$ during 40 cycles. We used the $2^{-\Delta \Delta \mathrm{Ct}}$ method that was based on the data from three biological replicates per treatment to calculate the relative gene-expression values. Ubiquitin (UBQ) genes were served as an internal control to normalize the sample variance (Chen et al., 2015). The experiments were conducted in triplicate, and the reaction of each gene contained three replicates. Analysis of variance (ANOVA) analysis by Graphpad Prism (GraphPad Software, La Jolla, CA) was applied for the statistics of the expression data.

Subcellular localization and AS-ODN of PbrMAPK13. The open reading frame (ORF) of PbrMAPK13 was amplified using a forward primer, 5'-AGACAATCCATACTGAACAGCTC-3' and reverse primer, 5'-ATGAAATCATTGGAGCTGAAAGAGAT-3'. The PCR product cut by the SpeI and BamHI enzymes (TaKaRa) was ligated into PUC19 digested with SpeI and BamHI to generate the CaMV35S:PbrMAPK13GFP (green fluorescent protein) construct, which was verified by sequencing. The CaMV35S:GFP construct was used as a control. The PbrMAPK13-GFP plasmid was then transformed into arabidopsis protoplasts, and GFP fluorescence was observed using a confocal laser-scanning microscope (DM2500; Leica, Beijing, China).

RNAstructure was used to predict the secondary structure of designated AS-ODNs, after which we designated antisense oligonucleotide primers in Oligo7.0 (Meng et al., 2014). Custom-made oligodeoxynucleotides [high-performance liquid chromatography-purified; Sigma-Genosys], native phosphodiester, and with phosphorothioate modifications in both the $5^{\prime}$ and $3^{\prime}$ terminals (three or four bases modified in each end) are indicated in lowercase: AS1 $\left(5^{\prime}-\operatorname{ccg}\right.$ TATCTCCGCTCCAGcct- $\left.3^{\prime}\right)$; AS2 (5'-gaaCTCCCTCTTTGAAGGTggc- $\left.3^{\prime}\right)$; AS3 (5' -gtaCTCGAGTGAACAGTGGAccg-3'); AS4 (5' ctgTTGGCACCCTCGGCGGcgg-3'); and AS5 (5' ctcCAGTGTTCCGGTGAGCAgct- $\left.3^{\prime}\right)$. To determine the efficiency of the AS-ODN-mediated gene knockdown, qPCR could be utilized. For each antisense oligonucleotide, the corresponding sense ODN is used as a negative control. Several variables were evaluated during growth in GM supplemented with a concentration range of cytofectin: 1, 10, 20, 30, 40, and $50 \mathrm{ng} \cdot \mathrm{mL}^{-1}$ (Moutinho et al., 2001). After evaluating the efficiency and toxicity of cytofectin in growing pollen tubes, a concentration of $30 \mathrm{ng} \cdot \mathrm{mL}^{-1}$ was used in the experiments, and a $4 \mathrm{~h}$ period was used to quantify the pollen-tube length
(Meurer-Grimes, 1995). The pollen-tube transfection and cultured methods were genotyped as described previously. We obtained the images of pollen-tube growth and pollengrain germination with a light microscope (BX51; Olympus). Three replications with 100 pollen grains were chosen randomly for measurement of the length of pollen tubes.

\section{Results}

Sequence identification of MAPKs genes in Rosaceae. To obtain a complete overview of the MAPKs gene family in Rosaceae, we conducted a genome-wide analysis using various bioinformatics resources. The MAPKs domain profile (PF07714) was used by hidden Markov model (HMM) to conduct a jackhammer to acquire the redundant MAPK genes of chinese white pear, apple, peach, strawberry, and plum, the five Rosaceae species (Eddy, 2011). We then used the arabidopsis MAPK protein sequences to perform repetitive BLAST against the candidate MAPK genes to eliminate the pseudoMAPK genes. Finally, we collected 87 MAPK sequences from five different Rosaceae species. Among them, a total of 23 MAPK genes were identified in chinese white pear (PbrMAPK), 15 in peach (PpeMAPK), 26 in apple (MdoMAPK), 11 in strawberry (FveMAPK), and 12 in plum (PmuMAPK), whose protein sequences harbored typical domains and motifs of MAPK proteins, S-TKc. Additionally, the predicted chinese white pear MAPK gene family sequences that were downloaded from the pear center database were constructed by our laboratory (Wu et al., 2013). The characteristics of the MAPK genes in the five Rosaceae species are listed in Table 1.

CONSERVED DOMAIN AND PHYLOGENETIC ANALYSIS OF MAPKS Gene Family in Rosaceae. To determine the phylogenic relationship of the MAPK family between chinese white pear and the other species of Rosaceae, an ML phylogenetic tree was constructed using full-length amino acid sequences by the RAxML program for phylogenetic analysis of large datasets (Stamatakis, 2006). AtBK3 from arabidopsis was used as the out-group. According to the topology and relative genetic distance of the tree, the MAPK gene family of five Rosaceae species and arabidopsis clustered into four distinct groups [A-D (Fig. 1)]. In addition, all Rosaceae MAPK genes that shared the family joint structure contained three highly conserved protein kinase domains (PROTEIN-KINASE-ATP, MAPK, and PROTEIN-KINASE-ST). Four groups were divided into two subtypes (type I and type II) by a TEY or TDY motif in their phosphorylation site (Pitzschke et al., 2009). The TDY type (type I) existed in groups $\mathrm{A}-\mathrm{C}$, whereas type II (group D) contained only the TEY motif. Interestingly, we found that a TDY motif-phosphorylation site from two apple genes existed in group D [MAPK25 and MAPK26 (Supplemental Fig. 1)]. Of the two subtypes, type I contained 31 MAPK genes and type II constituted the largest clade containing 63 MAPKs. For the 23 PbrMAPKs, group D possessed vast proportions of total members, including 13 genes; both groups $\mathrm{A}$ and $\mathrm{B}$ containing three chinese white pear genes and group $\mathrm{C}$ containing four chinese white pear genes (Fig. 1). According to the statistics, MAPK genes were evenly distributed in each subfamily.

The ORIGIN AND EXPANSION OF THE MAPK GENE FAMILY. Several gene-duplication modes drive the evolution of proteincoding gene families, including whole-genome duplication (WGD) or segmental duplication, tandem duplication, and 
Table 1. Genome information and MAPK genes subfamily identified in Rosaceae species in chinese white pear.

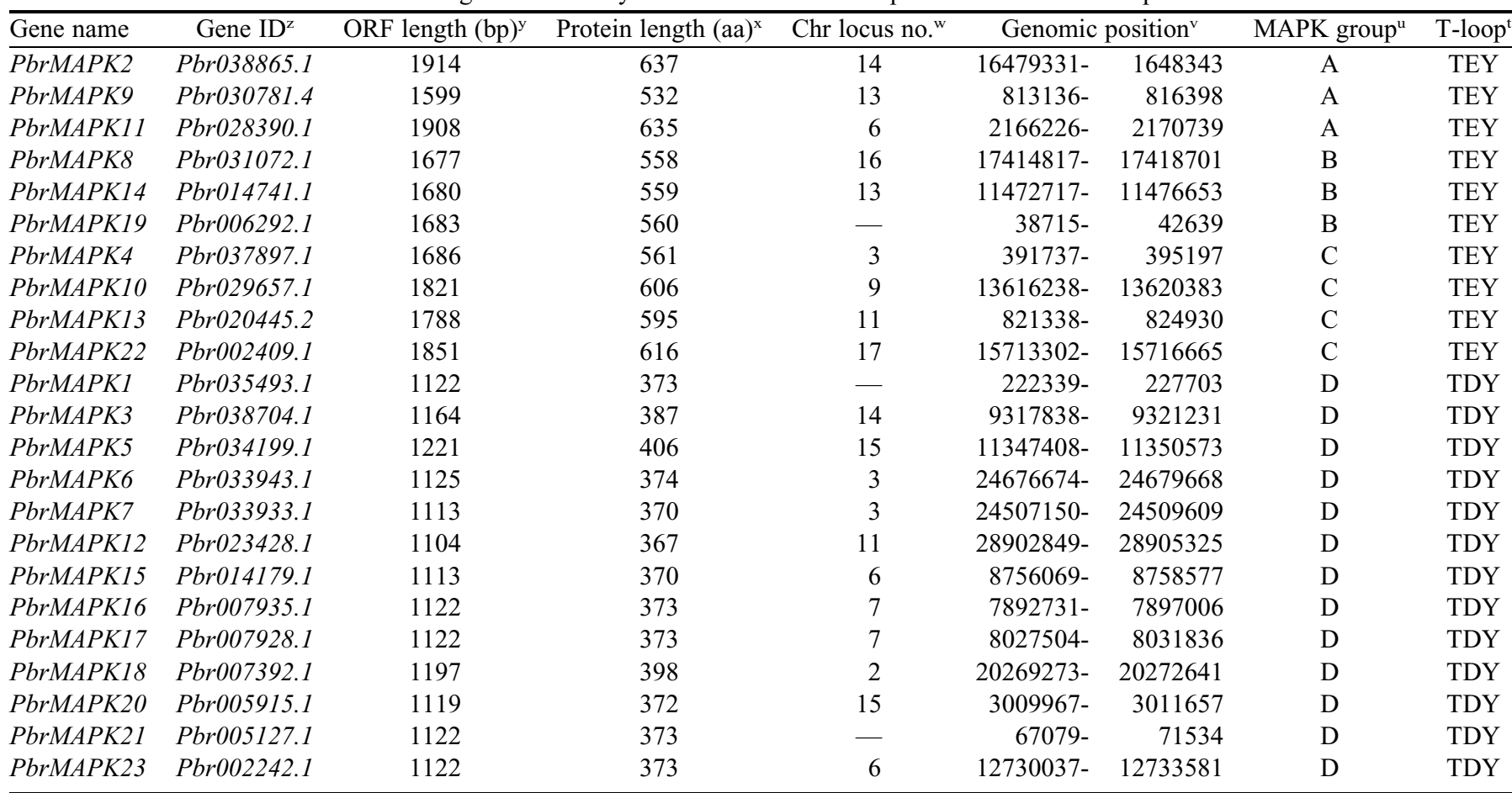

${ }^{\mathrm{z}}$ Gene identity from the chinese white pear database (Wu et al., 2013).

${ }^{y}$ Open reading frame.

${ }^{\mathrm{x}}$ Number of amino acids.

${ }^{\mathrm{w}}$ Chromosome locus number.

${ }^{\mathrm{v}}$ The start to end of genomic position.

${ }^{\mathrm{u}} \mathrm{A}-\mathrm{D}$ represent four subfamily.

${ }^{\mathrm{t}}$ Phosphorylation sites of MAPK.

rearrangement at the gene and chromosomal level. We detected the origins of duplicate genes for the MAPK genes family in five Rosaceae genomes with MCScanX. Each member of the MAPK gene family was classified into three types: WGD or segmental, proximal, and dispersed. Different patterns of gene duplication contributed differentially to the expansion of the MAPK gene family in the investigated taxa (Freeling and Thomas, 2006).

Remarkably, all MAPK genes in strawberry, peach, and chinese white pear were duplicated and retained from three duplication events, whereas MAPK genes only dispersed evenly in apple. Considering that the relationships of the five species were very close, we did not readily accept this result. Because MCScanX uses the locations of genes and their BLASTP results to recognize the duplication types of genes, we inferred that the genome annotation of apple might be not accurate. The WGD or segmental events in chinese white pear were in higher proportion than the other duplication events (Supplemental Fig. 2). Discussed in relation to the two subtypes, the proportions of dispersed MAPK gene duplication were considerably higher than those of WGD or segmental in the type II, and the type II had experienced proximal duplicates, whereas the type I did not. Proximal duplicates have different features and evolutionary fates than WGD or dispersed duplicates do; thus, the MAPK genes in type II may show a more specialized function than others. These results indicate that WGD or segmental duplication and dispersed gene duplication play critical roles in the expansion of the MAPK gene family in Rosaceae.

23 PbrMAPK genes were anchored on 11 chromosomes. Similar to PbrMAPK genes, the distribution of the MAPK genes in chromosomes of the other five Rosaceae genomes was random. Both segmental and tandem duplication events were analyzed to investigate the evolutionary mechanism of the five Rosaceae MAPK families. Seven segmental duplication events (14 genes) were identified in the chinese white pear MAPK family. Three were found in the group D and four in the other groups. Conserved synteny was observed in 27 of 34 MAPK regions in chinese white pear and strawberry, 28 of 35 regions in chinese white pear and plum, 29 of 38 regions in chinese white pear and peach, and 14 of the 23 regions in chinese white pear. Most MAPK genes were included in these regions. Segmental duplication event genes may exist in one chinese white pear chromosome with the other species chromosomes. Two tandem duplication clusters containing four genes were found on chromosomes 3 and 7 in chinese white pear: PbrMAPK6-PbrMAPK7 and PbrMAPK16-PbrMAPK17. No tandem MAPK gene duplications were identified in plum, and six tandem duplication clusters including 16 genes were identified in the apple MAPK family (Fig. 2).

To investigate the selection pressure acting on the MAPK genes in chinese white pear, we calculated the $\mathrm{Ka} / \mathrm{Ks}$ value of each gene pair from their respective groups. The $\mathrm{Ka} / \mathrm{Ks}$ values were used for measuring the selection pressure. It was found 


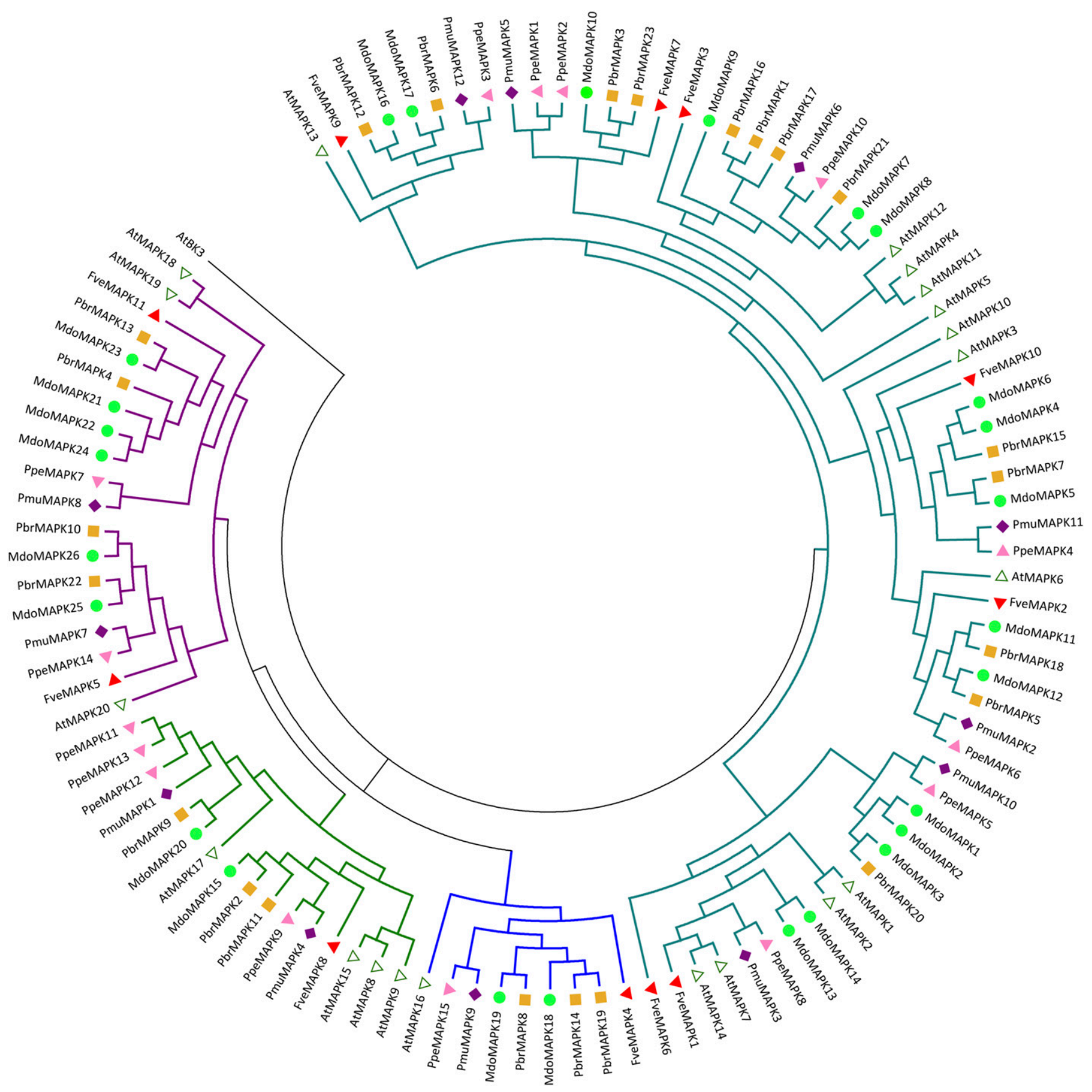

Fig. 1. Phylogenetic relationships between MAPK gene families from arabidopsis, chinese white pear, peach, apple, strawberry, and plum. MAPKs from chinese white pear species (PbrMAPKs) are highlighted by the yellow squares, and the other MAPKs from arabidopsis, apple, peach, strawberry, and plum are indicated by shapes of different colors as shown in the legend. Four subfamilies (A-D) are labeled by different colors and separated by black lines. MAPK $=$ mitogenactivated protein kinase.

that group $\mathrm{C}$ showed a higher $\mathrm{Ka} / \mathrm{Ks}$ value than the other groups. We concluded that genes in group $\mathrm{C}$ evolve much more divergence than the other groups, even though some gene pairs in group B had relatively high $\mathrm{Ka} / \mathrm{Ks}$ values. The evolution of MAPK genes in the other three groups was conserved compared with group $\mathrm{C}$, which demonstrated that most MAPK genes were functionally conversed (Fig. 3).

EXPRESSION PATTERNS OF MAPKS IN CHINESE WHITE PEAR. TO investigate the steady-state expression patterns of PbrMAPK genes, RT-PCR was used to determine the transcript levels in root, stem, leaf, style, pollen, and fruit. It was difficult to design primers because the sequences of PbrMAPK16, PbrMAPK17, and PbrMAPK1 were highly similar (Supplemental Fig. 3). As a result, the expression of PbrMAPK1 may have been similar to those of the other two genes. Individual PbrMAPKs exhibited different and overlapping patterns of expression.

To investigate which MAPK genes have similar functions and which gene is specifically expressed in pollen, we analyzed 

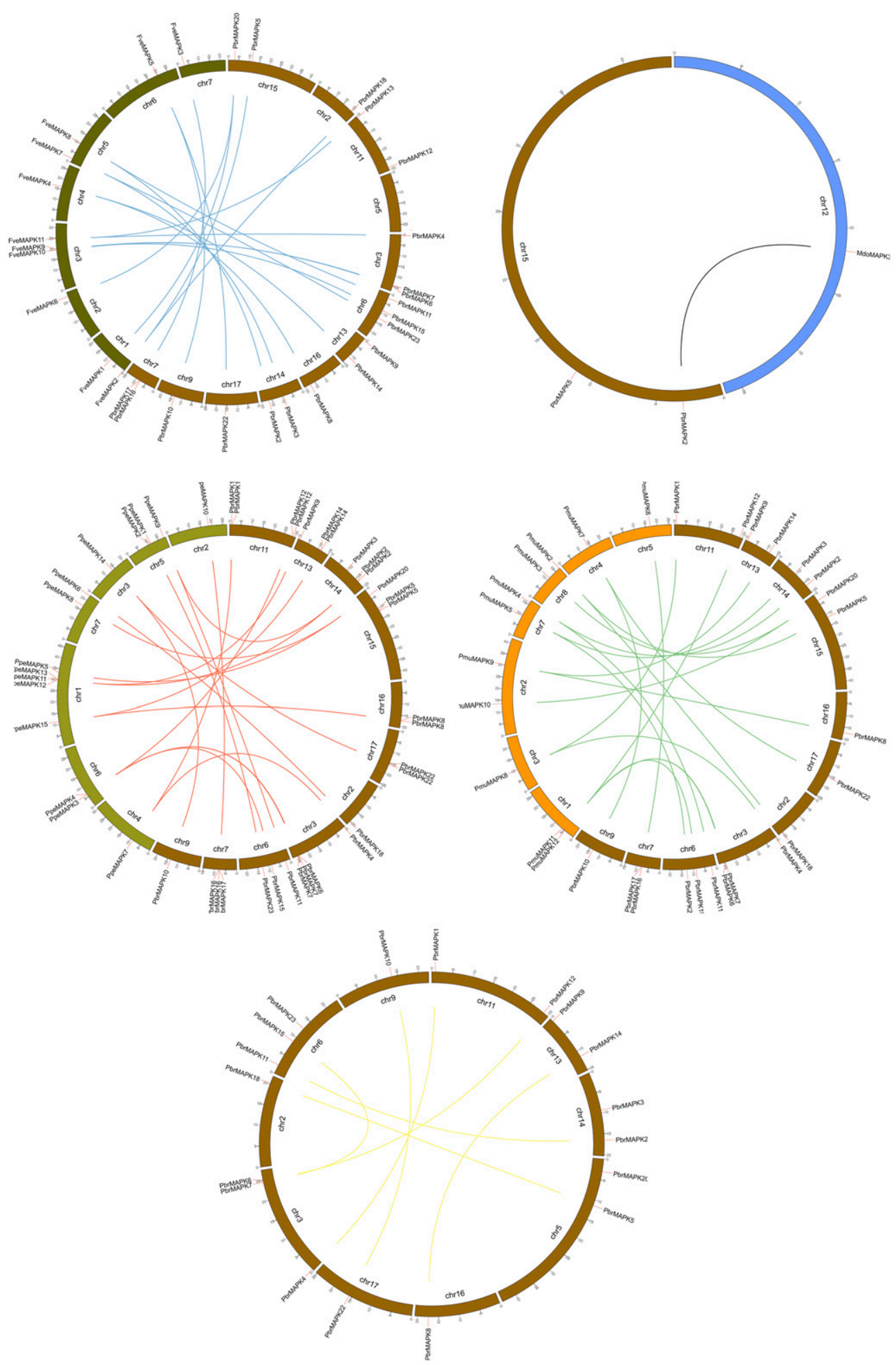

Fig. 2. MAPK genes in chinese white pear, peach, apple, strawberry, and plum were mapped on the different chromosomes. Chromosome or scaffold number is indicated on the inner side and highlighted using short red lines outside the circle corresponding to different MAPK genes. Gene pairs with a syntenic relationship are joined by the curves. MAPK = mitogen-activated protein kinase.

the tissue-specific expression patterns of paralogous MAPK genes in chinese white pear. The results revealed that PbrMAPK1, PbrMAPK8, PbrMAPK12, and PbrMAPK23 were expressed in all tissues except pollen, which had similar expression patterns in chinese white pear, although many of them appeared to contain quantitatively different mRNA levels of certain tissues. PbrMAPK7, PbrMAPK10, PbrMAPK13, PbrMAPK15, PbrMAPK18, and PbrMAPK20 had higher levels of pollen that might be related to pollen growth and development. In the sample organs, fruit showed the highest MAPK 

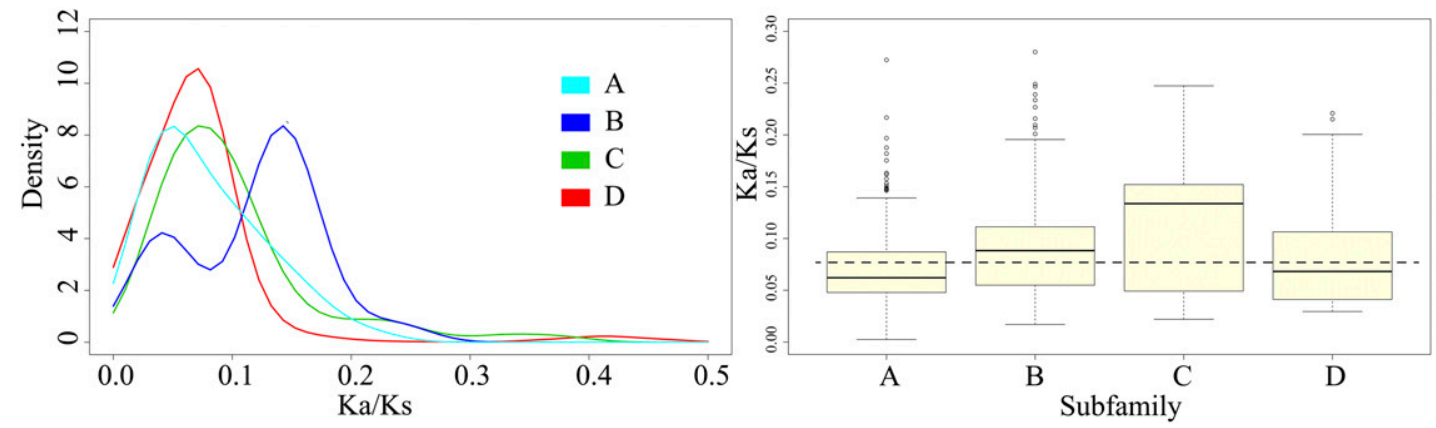

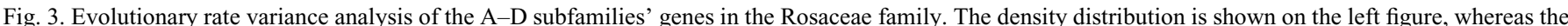
box-shaped figure is shown on the right. The significance test was performed by ANOVA $\left(P \leq 2.2 \mathrm{e}^{-16}\right)$; Ka $=$ nonsynonymous substitutions per site, Ks $=$ synonymous substitutions per site. ANOVA = analysis of variance.

gene expression. All genes in group $\mathrm{C}$ and many MAPKs of group D (PbrMAPK5, 7, 15, 18, 20, and 21) were widely expressed throughout all sample organs, suggesting that these MAPKs may be involved in the cellular activities related to basic life.

EXPRESSION PROFILES OF PBRMAPK RESPONSES TO VARIOUS STRESSES AND SIGNALS IN POLLEN. We analysed the expression pattern of PbrMAPKs by qPCR for pollen tubes in different temperatures and plant growth regulators treatment, and the total RNA extracted from pollen of the chinese white pear after $4 \mathrm{~h}$ of growth and subjected to temperature stress, BR, ABA, IAA, SPM, and SPD treatments. Primers used for RT-PCR analysis are listed in Table 2.

To explore the responses of MAPK genes to temperature stress, chinese white pear pollen was exposed to high or low temperatures (Fig. 4A and B). qPCR was then performed using the cDNAs obtained from the treated and untreated pollen as templates. The results of the qPCR for the temperature stress are shown in Fig. 4A. Compared with the control groups, the expression of 15 PbrMAPK genes significantly increased in a $37{ }^{\circ} \mathrm{C}$ high temperature stress. Only two PbrMAPKs (PbrMAPK1 and 3) were downregulated and the other four PbrMAPKs (PbrMAPK2, 4, 8, and 10) changed little. Among them, the expressions of PbrMAPK13 and PbrMAPK20 were the highest at $37{ }^{\circ} \mathrm{C}$. With a $17{ }^{\circ} \mathrm{C}$ treatment, the expression levels of four PbrMAPKs (PbrMAPK4, 13, 14, and 19) increased, and $P b r M A P K 13$ was also significantly upregulated.

In vitro germination of pollen of the five phytohormone treatment plants verified that BR, SPD, and SPM could promote the growth of pollen tubes (Supplemental Fig. 4). To explore the response of PbrMAPK genes to these signaling molecules, we conducted a qPCR analysis on treated and untreated chinese white pear pollen. The results of the qPCR analysis are shown in Fig. 4B. Twelve PbrMAPKs (PbrMAPK1, 2, 4, 8, 9, 10, 11, $13,19,21,22$, and 23) were significantly upregulated after BR treatment. Only three PbrMAPK genes (PbrMAPK15, 18, and

Table 2. List of PbrMAPKs, internal control genes primers, and PCR product length used for quantitative polymerase chain reaction gene expression.

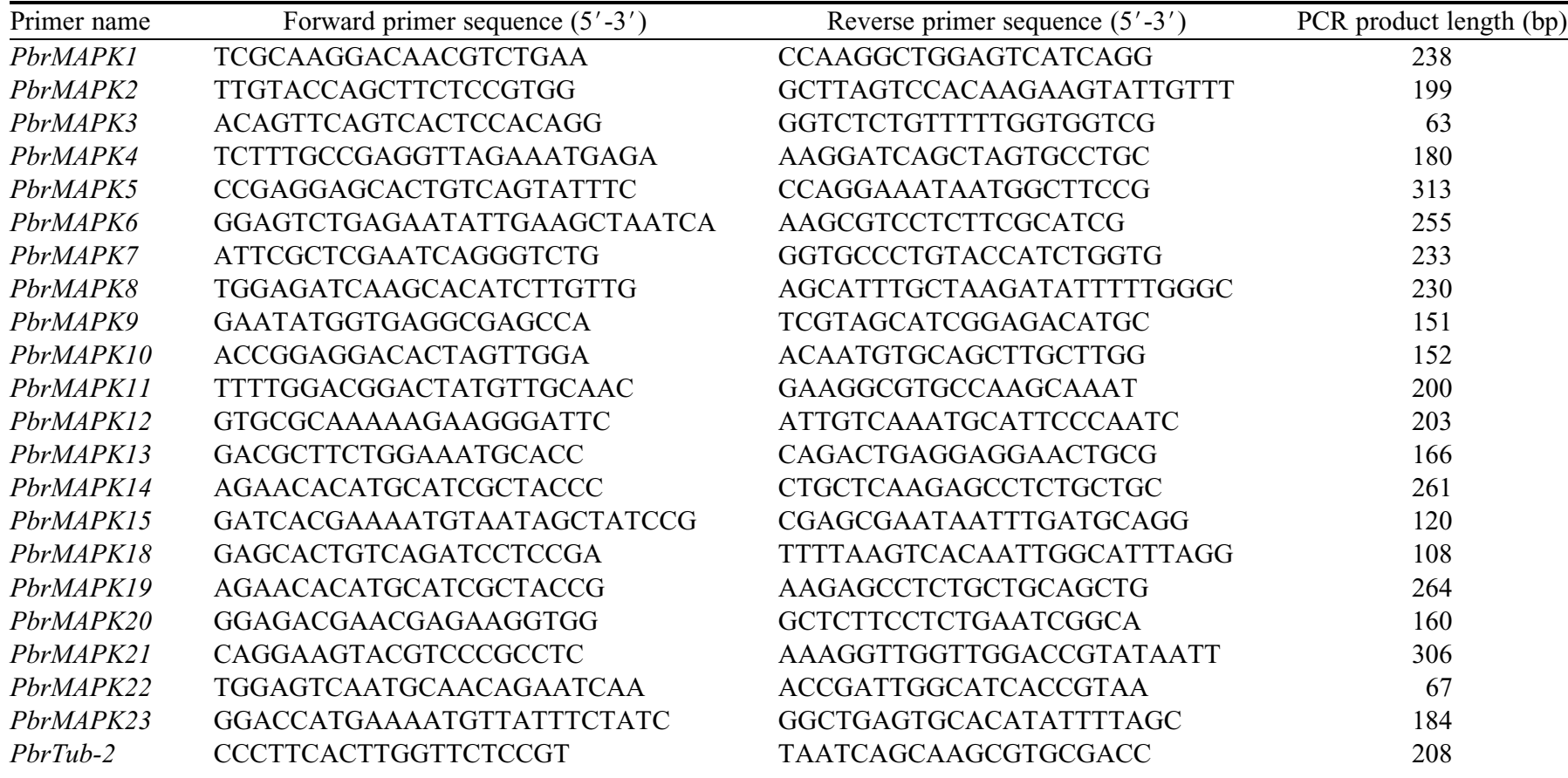



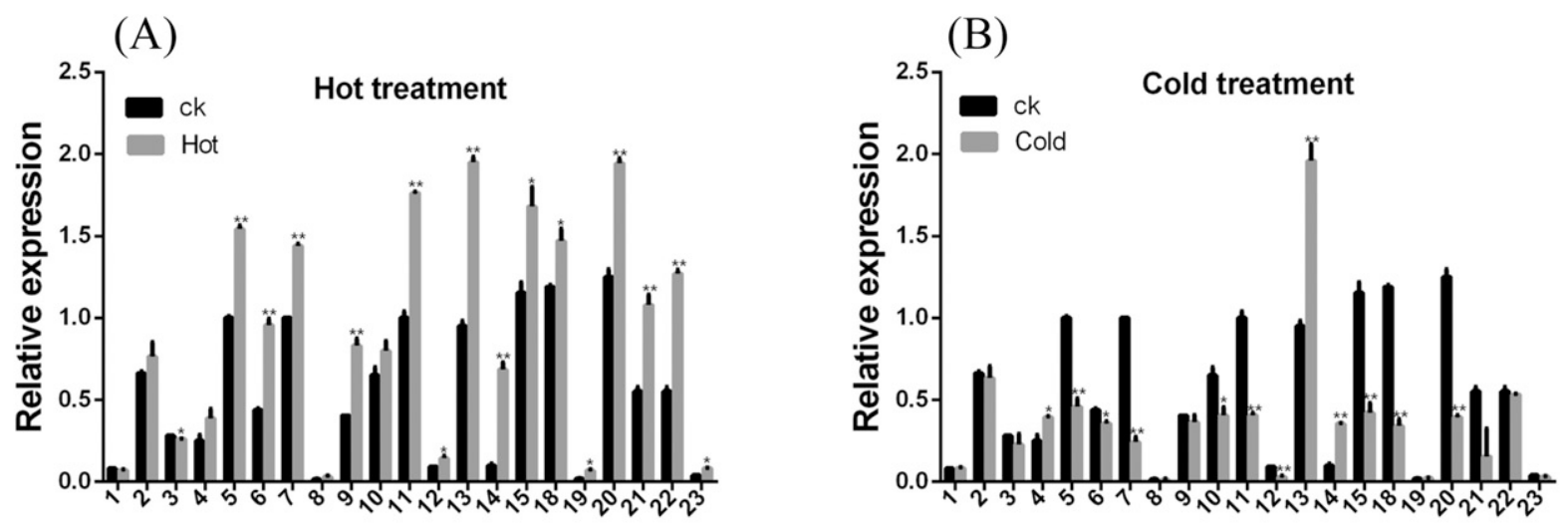

(C)

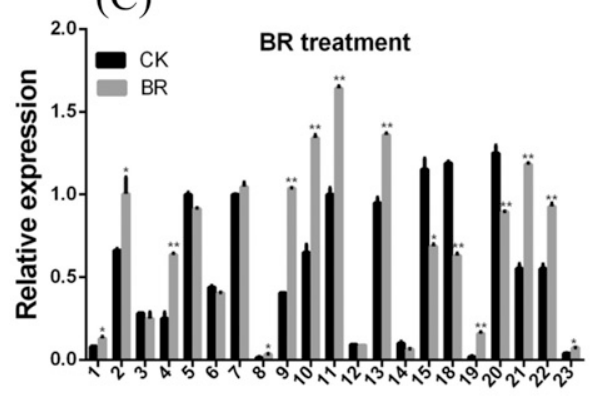

(D)

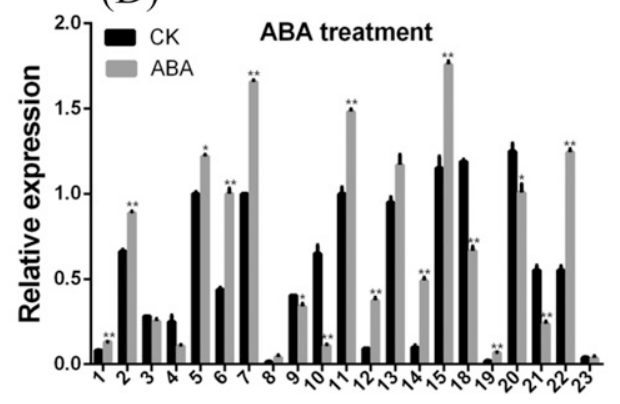

(E)

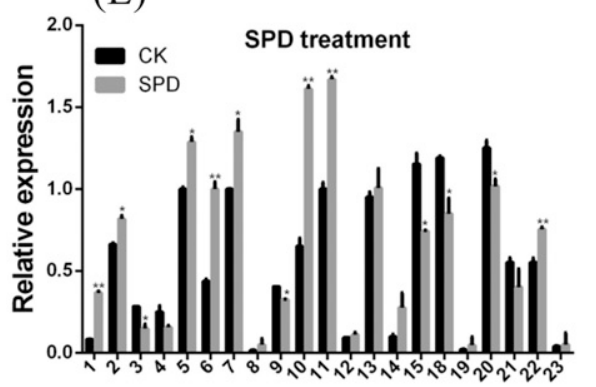

(F)

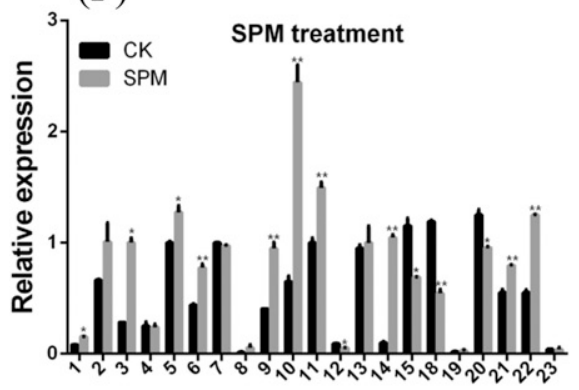

(G)

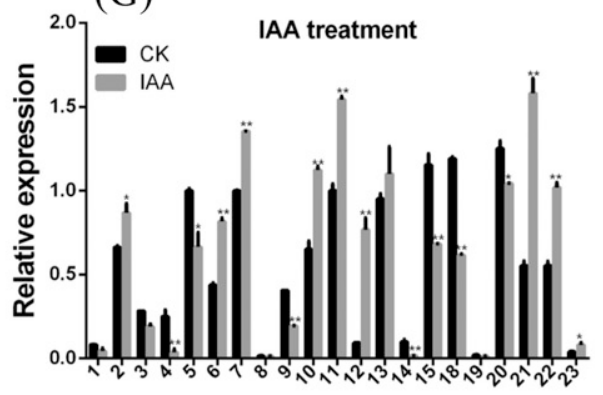

Fig. 4. Expression of chinese white pear MAPK genes (PbrMAPK) in pollen hydrated for $4 \mathrm{~h}$. The expression patterns of PbrMAPK genes under phytohormones (BR, ABA, IAA, SPM, and SPD) are depicted in $\mathbf{C}-\mathbf{G}$, and temperature stresses [cold $\left(17^{\circ} \mathrm{C}\right)$, hot $\left(37^{\circ} \mathrm{C}\right)$ ] are depicted in $\mathbf{A}$ and $\mathbf{B}$. Gene-expression analysis was performed by qPCR $4 \mathrm{~h}$ after each treatment and at $0 \mathrm{~h}$ for nontreated samples. The y-axis indicates the relative expression levels (in fold change) of treated vs. untreated (control) samples for each gene and the x-axis shows PbrMAPK genes. The error bars were calculated based on three biological replicates using SD. Independent-samples $t$ test was used to determine statistical differences; *indicates significant difference at $P \leq 0.05, * *$ indicates highly significant difference $P$ $\leq$ 0.01. $\mathrm{BR}=$ brassinosteroid; $\mathrm{ABA}=$ abscisic acid; IAA = indole-3-acetic acid; $\mathrm{SPM}=$ spermine; $\mathrm{SPD}=$ spermidine; $\mathrm{SD}=$ standard deviation.

20) were significantly downregulated. These results implied that $P b r M A P K 1,2,4,8,9,10,11,13,15,18,19,20,21,22$, and 23 may be involved in BR-dependent signaling pathways, and PbrMAPK3, 5, 6, 7, 12, and 14 may be involved in BRindependent signaling at all occurrences. After the pollen germination, $\mathrm{ABA}$ promoted the growth of pollen tubes, with the concentration increasing in a low-concentration range (10$\left.60 \mathrm{mg} \cdot \mathrm{L}^{-1}\right)$. Our results showed that eight out of the 16 chinese white pear genes were induced by ABA. The expression levels of PbrMAPKs (PbrMAPK9, 10, 18, 20, and 21) were significantly downregulated, whereas the other 11 PbrMAPKs were upregulated (Supplemental Table 1). SPD had the greatest impact on pollen-germination rate, whereas SPM was more favorable to pollen-tube growth (Meurer-Grimes, 1995; Wu et al., 2010). After the SPD and SPM treatment, expression levels of MAPK genes had similar trends, except for
PbrMAPK21. In general, most genes were upregulated by plant hormones. The MAPK genes showed different expression levels in different subtypes, depending on the treatment type, whereas the genes had a similar expression pattern in the same type. It has been proposed that they had similar functions of proteins classified in the same subtypes.

PbrMapK13 localization and Expression Pattern. To investigate the subcellular localization of PbrMAPK13, a transient transformation assay was conducted. When PbrMAPK13GFP was transformed into arabidopsis protoplasts, the signals were targeted mainly in the nucleus and plasma membrane, whereas the control GFP (CaMV35S:GFP) was distributed in both the cytoplasm and the nucleus (Fig. 5A).

The expression pattern of PbrMAPK13 was examined using qRT-PCR. The PbrMAPK13 transcript was ubiquitously expressed in all chinese white pear organs examined, with 
(A)

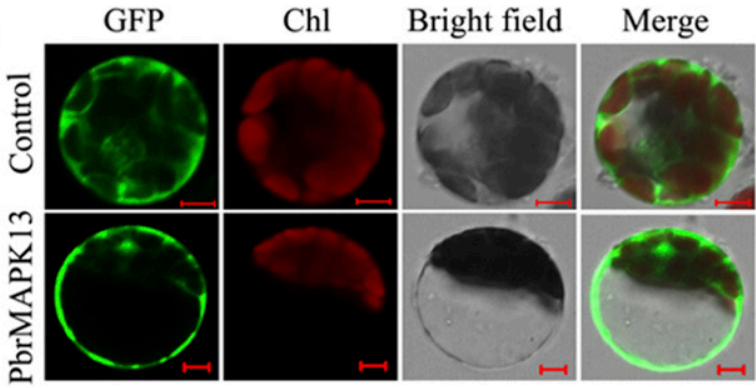

(B)

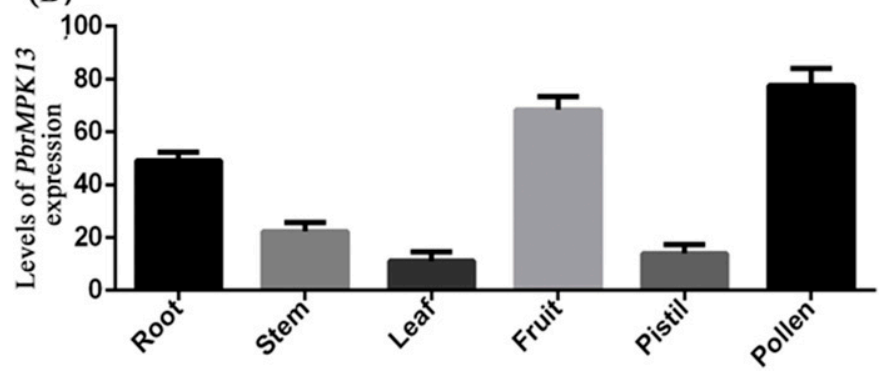

Fig. 5. Subcellular localization and the expression pattern in different tissues of PbrMAPK13. (A) Subcelluar location analysis of PbrMAPK13 in the arabidopsis protoplasts. PbrMAPK13 was shown to target the nucleus and plasma membrane by transient expression of PbrMAPK13-GFP (green fluorescent protein) in arabidopsis protoplasts. The upper line indicates the control, which showed 35S:GFP transformation in arabidopsis protoplasts. Chl refers to chloroplasts. (B) Expression of PbrMPK13 in different tissues is quantified by the percentage of reference gene, and the quantitative real-time polymerase chain reaction was repeated three times. UBQ genes were served as control. Bars show $\mathrm{SD}$. UBQ = ubiquitin; $\mathrm{SD}=$ standard deviation.

a relatively higher level in pollen. A relatively weak expression was observed in the leaf (Fig. 5B). We can speculate that PbrMAPK13 is involved in pollen growth and development.

The Study of PbrMAPK13 influence on POLlen growth BY AS-ODN. We designed phosphorothioated AS-ODN based on the specific region of PbrMAPK13 cDNA as described to downregulate PbrMAPK13 expression in the pollen tube. Five AS-ODNs were complementary to different sites of PbrMAPK13 mRNA, AS1, AS2, AS3, AS4, and AS5, and one sense oligodeoxynucleotide (S-ODN) was synthesized. Each was transfected into pollen cells by polysorbate 80 (PS80)-coated poly(n-butylcyano-acrylate) nanoparticles (Hamel et al., 2012). The results indicated that pollen-tube growth was inhibited when PbrMAPK13 had been silenced (Fig. 6A). AS5 was selected for the subsequent experiments. qPCR results indicated that the gene expression of PbrMAPK13 was significantly downregulated after the specific gene silencing mediated by AS5 (Fig. 6B). Pollen-germination experiments were performed at the temperature stress and showed that the mean lengths of the pollen tubes were lower in the plants treated with AS-ODN than control (CK) treated with S-ODN only. The length of pollen tubes in low temperature reduced much more significantly than that in normal and high temperatures (Fig. 6C). This result further suggested that PbrMAPK13 could respond to chilling stress.

\section{Discussion}

MAPK cascades have been implicated in regulating various stresses in plants, including signal transduction pathways, disease resistance, and responses to environmental stress $(\mathrm{Li}$ and Tax, 2013). In addition, the presence of MAPK genes in terrestrial plants ranging from mosses to eudicots makes them interesting candidates for studying the evolution of plant development (Hamel et al., 2012). To date, information related to pear MAPK genes has not been reported. In our study, we identified 23 PbrMAPKs based on the chinese white pear genome database. The MAPK gene family in chinese white pear is equivalent to apple (26 MdMAPKs) and is larger than that in other species. It can be speculated that the presence of more MAPK genes in chinese white pear and apple genomes may reflect considerable requirements for these genes to be involved in complicated transcriptional regulations in these woody perennial species.

A phylogenetic tree of the MAPK family proteins from arabidopsis, chinese white pear, peach, apple, strawberry, and plum was constructed using multiple-sequence alignment. The phylogenetic tree divided the MAPK proteins into four subgroups labeled A-D on the basis of previous reports. Gene duplications play an important role not only in the genomic rearrangement and expansion but also in diversification of gene
(A)

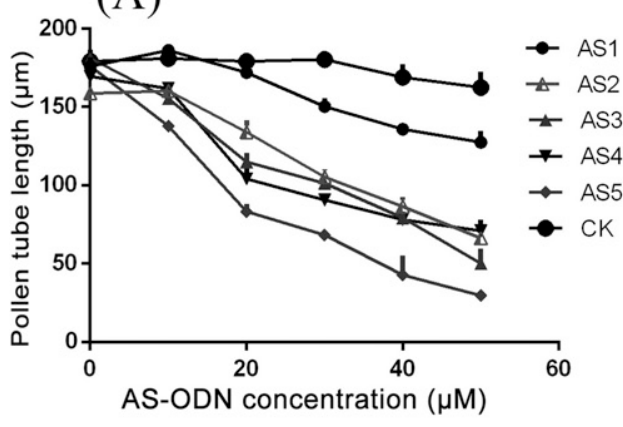

(B)

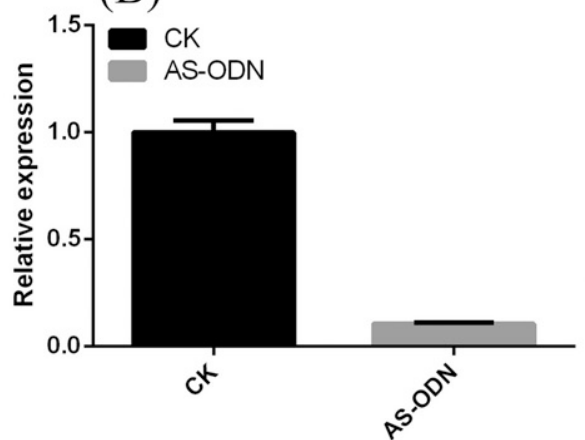

(C)

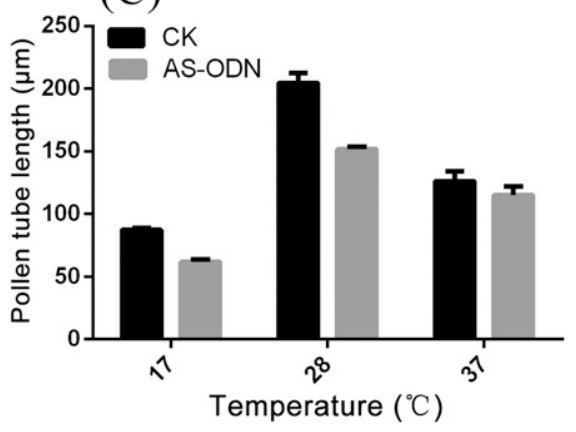

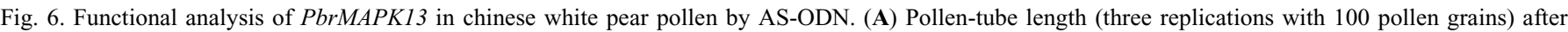
treatment with five AS-ODNs (AS1, AS2, AS3, AS4, and AS5) and one-sense oligodeoxynucleotide [S-ODN (CK = control)] for PbrMAPK13 in different concentrations $(0,10,20,30,40$, and $50 \mu \mathrm{M})$. (B) qPCR analysis of PbrMAPK13 expression in S-ODN (CK) and AS5 transfected chinese white pear pollen after 4 h. (C) Quantitative comparison of pollen-tube length of low $\left(17^{\circ} \mathrm{C}\right)$, high $\left(37^{\circ} \mathrm{C}\right)$, and normal temperatures after AS-ODN and S-ODN treatment for $4 \mathrm{~h}$ in a concentration $30 \mu \mathrm{M}$. qPCR was repeated three times. Bars show SD. AS-ODN = antisense oligodeoxynucleotide; qPCR $=$ quantitative polymerase chain reaction; $\mathrm{SD}=$ standard deviation. 
function (Guan et al., 2014), and the results suggest that MAPK genes in these four species may have originated from a common ancestor (Wang et al., 2012b). In the present study, chromosomal location analysis showed that MAPK genes of chinese white pear were dispersed throughout 11 out of 17 chromosomes with different densities. Two sister-pair PbrMAPKs were tightly colocated in the chinese white pear genome, whereas seven sister-pair PbrMAPKs were located on different chromosomes. By evolutionary rate variance analysis of the four A-D subfamilies, we determined that all the $\mathrm{Ka} / \mathrm{Ks}$ ratios of paralogous genes were less than one. This result suggests that MAPK genes have evolved mainly under the influence of purifying selection (Ganko et al., 2007).

The functional diversification of MAPK genes has been observed in several plant species, but few studies have related to their function in pollen. Since paralogous genes in the same genome were created by gene-duplication events and usually had similar functions (Zhang, 2003), and gene-expression patterns can provide important clues for gene function, we examined the expression of all the PbrMAPK genes in root, stem, leaf, style, pollen, and fruit tissues using semiquantitative RT-PCR. Given that our work focused on the role of MAPK in the regulation of the growth of pollen tubes, we were interested in the expression levels of genes encoding MAPK in various plant organs such as pollen. Interestingly, different groups had different expression patterns. In general, all MAPKs of Group A (PbrMAPK2, 9, and 11) and C (PbrMAPK4, 10, 13, and 22), most MAPKs of Group D (PbrMAPK5, 6, 7, 15, 18, 20, 21, and $23)$, and PbrMAPK14 in Group B were expressed in pollen (Supplemental Fig. 3). Overall, these data showed that many PbrMAPK genes are involved in the pollen growth, indicating that MAPK genes participating in the male gamete play a key role in plant sexual reproduction. The results suggest that geneduplication events are important in the gene-family evolution, for the duplicated genes provide the raw materials for the new features, which in turn guarantee the stability of gene function and facilitate the generation of new functions (Zhao, 2003). All these genes displayed diverse expression patterns, representing distinct roles of each PbrMAPK.

Plant MAPK genes play an important role in response to various abiotic and phytohormones of plant growth and development (Singh and Jwa, 2013; Xu and Zhang, 2015). Many studies have indicated that phytohormones and plant signaling molecules (BR, ABA, IAA, SPD, and SPM) are involved in plant environmental stress and pathogen resistance signaling pathways (Pitzschke et al., 2009). For instance, the BR signaling has also regulated plant stomatal development (Wang et al., 2007). At present, most molecular biology studies and reports focused on type II, in which genes harbored a TEY signature. For example, MAPK cascades, in particular those dependent on type II MAPK3/MAPK6, have been shown to be important regulators in response to plant hormones (Danquah et al., 2014) and participants in pollen-tube guidance (Guan et al., 2014), stomatal complex differentiation, and seed or root development (Lopez-Bucio et al., 2014). Since AtMAPK3 and AtMAPK6 participated in more than one biological process, we observed that PbrMAPK5, PbrMAPK7, PbrMAPK15, and PbrMAPK18 presented similar multifunction roles in the biological processes of pollen. PbrMAPK5, PbrMAPK7 may be participated temperatures, ABA, SPD, IAA treatments; PbrMAPK15, PbrMAPK18 participated in all the treatments in our studies (Supplemental Table 1).
Previous research has shown that AtMAPK4 participates in environmental stress responses, cell division, and disease resistance (Kosetsu et al., 2010). It also plays an important role in abiotic stress responses. MAPK9 and MAPK12 from arabidopsis have also been shown to participate in biotic stress responses and stomata closure. Similarly, in chinese white pear pollen, PbrMAPK5, PbrMAPK6, PbrMAPK7, and PbrMAPK20 from type II were also induced by high-/lowtemperature stress and phytohormones, which suggested that they may be involved in abiotic stress responses. We explored the evolutionary relationship between these four genes by Ks values. It was found that the divergent time of these genes occurred early before the evolution of chinese white pear (Supplemental Table 2), whereas the recent duplicated genes of these four genes were not found to be related to these stresses. The function of genes is determined by their coding DNA sequence (CDS) and cis-elements. Our research suggested that cis-elements play more important roles in the expression patterns of MAPK genes. For example, PbrMAPK5 and $\operatorname{PbrMAPK} 18$ were $93 \%$ similar in sequences, and they became divided recently (Fig. 1; Supplemental Table 2), whereas their expression patterns were distinct under our treatments. PbrMAPK15 nucleotide sequence had 58\% similarities with PbrMAPK20 but they shared similar expression patterns; however, their divergence occurred at a more ancient period. It was suggested that MAPK genes generated by duplication events could derive more functions, and even their sequences are extremely analog.

In type I genes, the activation site for encoding the amino acid sequences was the TDY motif, and the functions have been mentioned in only a few studies. Treatment with ABA can induce the transcriptional regulation of MAPK genes in arabidopsis (Danquah et al., 2014). Recently, MPK9, a type I MAPK in arabidopsis that is highly homologous with PbrMAPK2, was found to be preferentially expressed in guard cells and was upregulated downstream of the reactive oxygen species in guard cells' ABA signaling (Jammes et al., 2009; Singh and Jwa, 2013). PbrMAPK2, PbrMAPK11, and PbrMAPK22 in type I have similar expression patterns under various stress treatments, and all were highly induced by ABA. Our research also found that PbrMAPK1, PbrMAPK5, PbrMAPK6, PbrMAPK7, PbrMAPK10, PbrMAPK12, PbrMAPK14, PbrMAPK15, PbrMAPK18, PbrMAPK19, and PbrMAPK21 respond to the ABA stress (Supplemental Table 1). Furthermore, a close relationship was detected between AtMAPK3, PbrMAPK7, and PbrMAPK15;AtMAPK7 and PbrMAPK20; AtMAPK15, PbrMAPK2, and PbrMAPK11; and AtMAPK18, PbrMAPK4, and PbrMAPK13 (Fig. 1). This suggested that the gene-duplication events could generate more genes to respond to the same stress, although the expression pattern or activation might be altered. Interestingly, the type I gene, PbrMAPK13, showed almost no response to the phytohormone stimuli; however, it was significantly upregulated in temperature stress, which is similar to the case for MnMAPK1 in mulberry type I MAPKs (Wei et al., 2014). When PbrMAPK13 knockdown was mediated by the AS-ODNs under temperature stress, the growth length of pollen tubes became shorter than that of the control, especially with low-temperature treatment. PbrMAPK13 was found to be collinear with Pmu$M A P K 8, F v e M A P K 11$, and PpeMAPK7, which was evolutionary conserved. By $\mathrm{Ks}$ values of gene pairs between PbrMAPK13 and other PbrMAPKs, we inferred that 
PbrMAPK13 was duplicated by PbrMAPK4. Although their divergent times were not similar, the collinearity of the two genes suggested that they evolved conserved. Above all, the expression patterns of the two genes were similar, suggesting that the two genes are both functionally important for plants. Considered collectively, these results indicate that PbrMAPK13 played a major role in the development of pollen.

In summary, our results indicate that PbrMAPKs may play vital roles in biotic and abiotic stress responses in chinese white pear pollen. For example, PbrMAPK13 responded to hormone and temperature stresses by changed expression pattern, thus affected the development of pollen tube growth. Further studies into PbrMAPK functions and signal-transduction mechanisms need to be conducted. The transgenic approach is a good method for studying the functions of PbrMAPK genes, and we will continue to use these approaches in the future.

\section{Conclusions}

A total of 87 full-length MAPK genes were identified in the five Rosaceae genomes, and the chinese white pear genome contained 23 MAPK genes. According to the structural characteristics of the proteins determined through phylogenetic analysis, the MAPK genes were grouped into two types and four classes (A-D). Further expression-profile analysis using qPCR showed that most of the MAPK genes from chinese white pear pollen were induced by various stresses (high/low temperature) and phytohormones (BR, ABA, IAA, SPM, and SPD). On the usage of AS-ODNs in chinese white pear pollen, a more detailed study of PbrMAPK13 in response to temperature was possible. This new information provides some insights into new potential functions of PbrMAPKs, which provides the basis for further characterization of the physiological functions of PbrMAPKs.

\section{Literature Cited}

Abramoff, M.D., P.J. Magalhaes, and S.J. Ram. 2004. ImageJ processing with Image J. Biophoton. Intl. 11:36-42.

Altschul, S.F., W. Gish, W. Miller, E.W. Myers, and D.J. Lipman. 1990. Basic local alignment search tool. J. Mol. Biol. 215:403-410. Bateman, A., L. Coin, R. Durbin, R.D. Finn, V. Hollich, A.K. Sam Griffths-Jones, A. Khanna, M. Marshall, S. Moxon, E.L.L. Sonnhammer, D.J. Studholme, C. Yeats, and S.R. Eddy. 2004. The Pfam protein families database. Nucleic Acids Res. 32:138-141.

Chen, J., H. Yin, J. Gu, L. Li, Z. Liu, X. Jiang, H. Zhou, S. Wei, S. Zhang, and J. Wu. 2015. Genomic characterization, phylogenetic comparison and differential expression of the cyclic nucleotide-gated channels gene family in pear (Pyrus bretchneideri Rehd.). Genomics 105:39-52.

Colcombet, J. and H. Hirt. 2008. Arabidopsis thaliana MAPKs: A complex signalling network involved in multiple biological processes. Biochem. J. 413:217-226.

Danquah, A., A. Zelicourt, J. Colcombet, and H. Hirt. 2014. The role of $\mathrm{ABA}$ and MAPK signaling pathways in plant abiotic stress responses. Biotechnol. Adv. 32:40-52.

Eddy, S.R. 2011. Accelerated profile HMM searches. PLOS Comput. Biol. 7(10):1-16.

Edgar, R.C. 2004. MUSCLE: Multiple sequence alignment with high accuracy and high throughput. Nucleic Acids Res. 32:1792-1797.

Fan, L.M., H. Wang, and W.H. Wu. 2001. In vitro arabidopsis pollen germination and characterization of the inward potassium currents in arabidopsis pollen grain protoplasts. J. Expt. Bot. 361:1603-1614.

Freeling, M. and B.C. Thomas. 2006. Gene-balanced duplications, like tetraploidy, provide predictable drive to increase morphological complexity. Genome Res. 16:805-814.
Ganko, E.W., B.C. Meyers, and T.J. Vision. 2007. Divergence in expression between duplicated genes in arabidopsis. Mol. Biol. Evol. 24:2298-2309.

Garcia-Hernandez, M., T.Z. Berardini, G.H. Chen, D. Crist, A. Doyle, E. Huala, E. Knee, M. Lambrecht, N. Miller, L.A. Mueller, S. Mundodi, L. Reiser, S.Y. Rhee, R. Scholl, J. Tacklind, D.C. Weems, Y.H. Wu, I. Xu, D. Yoo, J. Yoon, and P.F. Zhang. 2002. TAIR: A resource for integrated arabidopsis data. Funct. Integr. Genomics 2:239-253.

Guan, Y., J. Lu, J. Xu, B. McClure, and S. Zhang. 2014. Two mitogenactivated protein kinases, MPK3 and MPK6, are required for funicular guidance of pollen tubes in arabidopsis. Plant Physiol. 165:528-533.

Hamel, L.P., M.C. Nicole, S. Duplessis, and B.E. Ellis. 2012. Mitogen-activated protein kinase signaling in plant-interacting fungi: Distinct messages from conserved messengers. Plant Cell 24:1327-1351.

Hord, C.L., Y.J. Sun, L.J. Pillitteri, K.U. Torii, H. Wang, S. Zhang, and H. Ma. 2008. Regulation of arabidopsis early anther development by the mitogen-activated protein kinases, MPK3 and MPK6, and the ERECTA and related receptor-like kinases. Mol. Plant 1:645-658.

Ichimura, K. 2002. Mitogen-activated protein kinase cascades in plants: A new nomenclature. Trends Plant Sci. 7:302-308.

Jammes, F., C. Song, D. Shin, S. Munemasa, K. Takeda, D. Gu, D. Cho, S. Lee, R. Giordoa, S. Sritubtim, N. Leonhardt, B.E. Ellis, Y. Murata, and J.M. Kwak. 2009. MAP kinases MPK9 and MPK12 are preferentially expressed in guard cells and positively regulate ROS-mediated ABA signaling. Proc. Natl. Acad. Sci. USA 106:20520-20525.

Kosetsu, K., S. Matsunaga, H. Nakagami, J. Colcombet, M. Sasabe, T. Soyano, Y. Takahashi, H. Hirt, and Y. Machida. 2010. The MAP kinase MPK4 is required for cytokinesis in Arabidopsis thaliana. Plant Cell 22:3778-3790.

Lee, T.H., H.B. Tang, X.Y. Wang, and A.H. Paterson. 2013. PGDD: A database of gene and genome duplication in plants. Nucleic Acids Res. 41:1152-1158.

Li, J. and F.E. Tax. 2013. Receptor-like kinases: Key regulators of plant development and defense. J. Integr. Plant Biol. 55:1184-1187.

Liu, Q. and Q. Xue. 2007. Computational identification and phylogenetic analysis of the MAPK gene family in Oryza sativa. Plant Physiol. Biochem. 45:6-14.

Liu, S., L. Hua, S. Dong, H. Chen, X. Zhu, J. Jiang, F. Zhang, Y. Li, X. Fang, and F. Chen. 2015. OsMAPK6, a mitogen-activated protein kinase, influences rice grain size and biomass production. Plant J. 84:672-681.

Lopez-Bucio, J.S., J.G. Dubrovsky, J. Raya-Gonzalez, Y. UgartecheaChirino, J. Lopez-Bucio, L.A. de Luna-Valdez, M. Ramos-Vega, P. Leon, and A.A. Guevara-Garcia. 2014. Arabidopsis thaliana mitogen-activated protein kinase 6 is involved in seed formation and modulation of primary and lateral root development. J. Expt. Bot. 65:169-183.

Meng, D., Z.Y. Gu, W. Li, A.D. Wang, H. Yuan, Q. Yang, and T.Z. Li. 2014. Apple MdABCF assists in the transportation of S-RNase into pollen tubes. Plant J. 78:990-1002.

Meng, X., H. Wang, Y. He, Y. Liu, J.C. Walker, K.U. Torii, and S. Zhang. 2012. A MAPK cascade downstream of ERECTA receptorlike protein kinase regulates arabidopsis inflorescence architecture by promoting localized cell proliferation. Plant Cell 24:4948-4960. Meurer-Grimes, B. 1995. New evidence for the systematic significance of acylated spermidines and flavonoids in pollen of higher Hamamelidae. Brittonia 47:130-142.

Moutinho, A., L. Camacho, A. Haley, M.S. Pais, A. Trewavasand, and R. Malhó. 2001. Antisense perturbation of protein function in living pollen tubes. Sex. Plant Reprod. 14:101-104.

Nicholas, K.B., H.B. Nicholas, and D.W. Deerfield, II. 1996. GeneDoc: Analysis and visualization of genetic variation. Embnew News 4:28-30.

Pitzschke, A., A. Schikora, and H. Hirt. 2009. MAPK cascade signalling networks in plant defence. Plant Biol. 12:421-426. 
Qiao, X., M. Li, L. Li, H. Yin, J.Y. Wu, and S.L. Zhang. 2015. Genome-wide identification and comparative analysis of the heat shock transcription factor family in chinese white pear (Pyrus bretschneideri) and five other Rosaceae species. BMC Plant Biol. 15:12-28.

Robinson, M.J. and M.H. Cobb. 1997. Mitogen-activated protein kinase pathways. Curr. Opin. Cell Biol. 9:180-186.

Singh, R. and N.S. Jwa. 2013. The rice MAPKK-MAPK interactome: The biological significance of MAPK components in hormone signal transduction. Plant Cell Rpt. 32:923-931.

Stamatakis, A. 2006. Raxml-vi-hpc: Maximum likelihood-based phylogenetic analyses with thousands of taxa and mixed models. Bioinformatics 22:2688-2690.

Wang, H., N. Ngwenyama, Y. Liu, J.C. Walker, and S. Zhang. 2007. Stomatal development and patterning are regulated by environmentally responsive mitogen-activated protein kinases in arabidopsis. Plant Cell 19:63-73.

Wang, Y., H. Tang, J.D. Debarry, X. Tan, J. Li, X. Wang, T.H. Lee, H. Jin, B. Marler, H. Guo, J.C. Kissinger, and A.H. Paterson. 2012a. MCScanX: A toolkit for detection and evolutionary analysis of gene synteny and collinearity. Nucleic Acids Res. 40:49-53.

Wang, Y., X. Wang, and A.H. Paterson. 2012b. Genome and gene duplications and gene expression divergence: A view from plants. Ann. N. Y. Acad. Sci. 1256:1-14.

Wei, C., X. Liu, D. Long, Q. Guo, Y. Fang, C. Bian, D. Zhang, Q. Zeng, Z. Xiang, and A. Zhao. 2014. Molecular cloning and expression analysis of mulberry MAPK gene family. Plant Physiol. Biochem. 77:108-116.
Wu, J., Z. Shang, J. Wu, X. Jiang, P.N. Moschou, W. Sun, K.A. Roubelakis-Angelakis, and S. Zhang. 2010. Spermidine oxidasederived $\mathrm{H}_{(2)} \mathrm{O}_{(2)}$ regulates pollen plasma membrane hyperpolarization-activated $\mathrm{Ca}^{(2+)}$-permeable channels and pollen tube growth. Plant J. 63:1042-1053.

Wu, J., Z. Wang, Z. Shi, S. Zhang, R. Ming, S. Zhu, M.A. Khan, S. Tao, S.S. Korban, H. Wang, N.J. Chen, T. Nishio, X. Xu, L. Cong, K. Qi, X. Huang, Y. Wang, X. Zhao, C. Deng, C. Gou, W. Zhou, H. Yin, G. Qin, Y. Sha, Y. Tao, H. Chen, Y. Yang, Y. Song, D. Zhan, J. Wang, L. Li, M. Dai, C. Gu, D. Shi, X. Wang, H. Zhang, L. Zeng, D. Zheng, C. Wang, M. Chen, G. Wang, L. Xie, V. Sovero, S. Sha, W. Huang, M. Zhang, J. Sun, L. Xu, Y. Li, X. Liu, Q. Li, J. Shen, R.E. Paull, and J.L. Bennetzen. 2013. The genome of the pear (Pyrus bretschneideri Rehd.). Genome Res. 23:396-408.

$\mathrm{Xu}, \mathrm{J}$. and S. Zhang. 2015. Mitogen-activated protein kinase cascades in signaling plant growth and development. Trends Plant Sci. 20:5664.

Zhang, J. 2003. Evolution by gene duplication: An update. Trends Ecol. Evol. 18:292-298.

Zhang, S., R. Xu, X. Luo, Z. Jiang, and H. Shu. 2013. Genome-wide identification and expression analysis of MAPK and MAPKK gene family in Malus domestica. Gene 531:377-387.

Zhang, T., Y. Liu, T. Yang, L. Zhang, S. Xu, L. Xue, and L. An. 2006. Diverse signals converge at MAPK cascades in plant. Plant Physiol. Biochem. 44:274-283.

Zhao, D. 2003. Members of the arabidopsis-SKP1-like gene family exhibit a variety of expression patterns and may play diverse roles in arabidopsis. Plant Physiol. 133:203-217. 


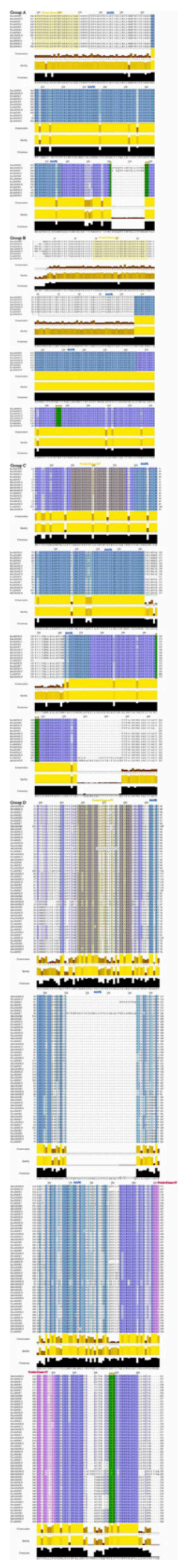

Supplemental Fig. 1. Protein sequence multi-alignment of the MAPKs from four subfamilies (A-D) of chinese white pear, peach, apple, strawberry, and plum. The names of subdomains are indicated with different colors words on the top of each row. The TXY phosphorylation sites are indicated by yellow asterisks. 


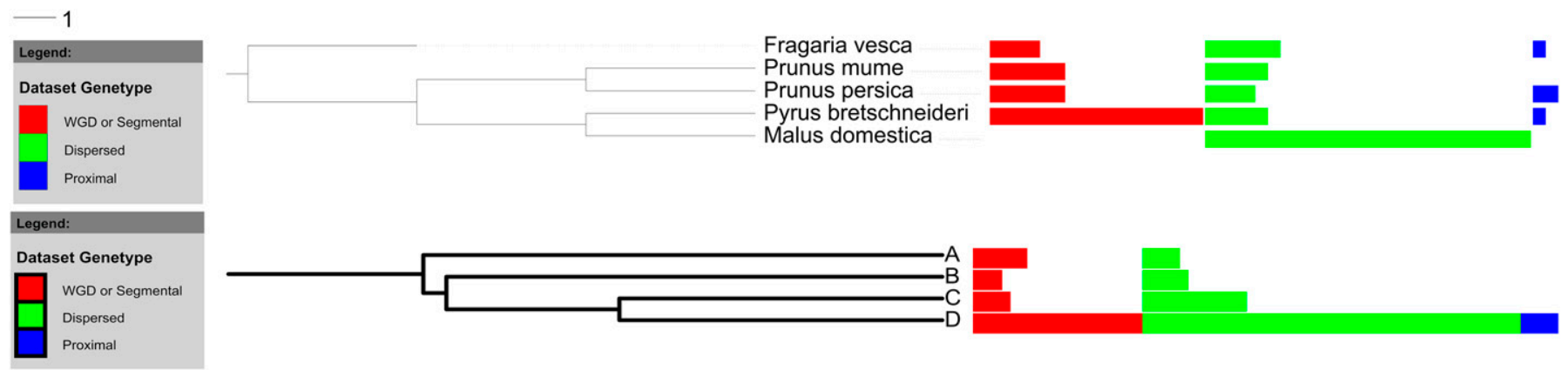

Supplemental Fig. 2. The distribution of gene duplications events in four subfamilies (A-D) of chinese white pear, peach, apple, strawberry, and plum. The color block length indicates that the duplications events corresponding to the proportion. Red blocks refer to whole-genome duplication (WGD) or segmental; green blocks refer to dispersed; blue blocks refer to proximal. 
Root Stem Leaf Pistil Pollen Fruit

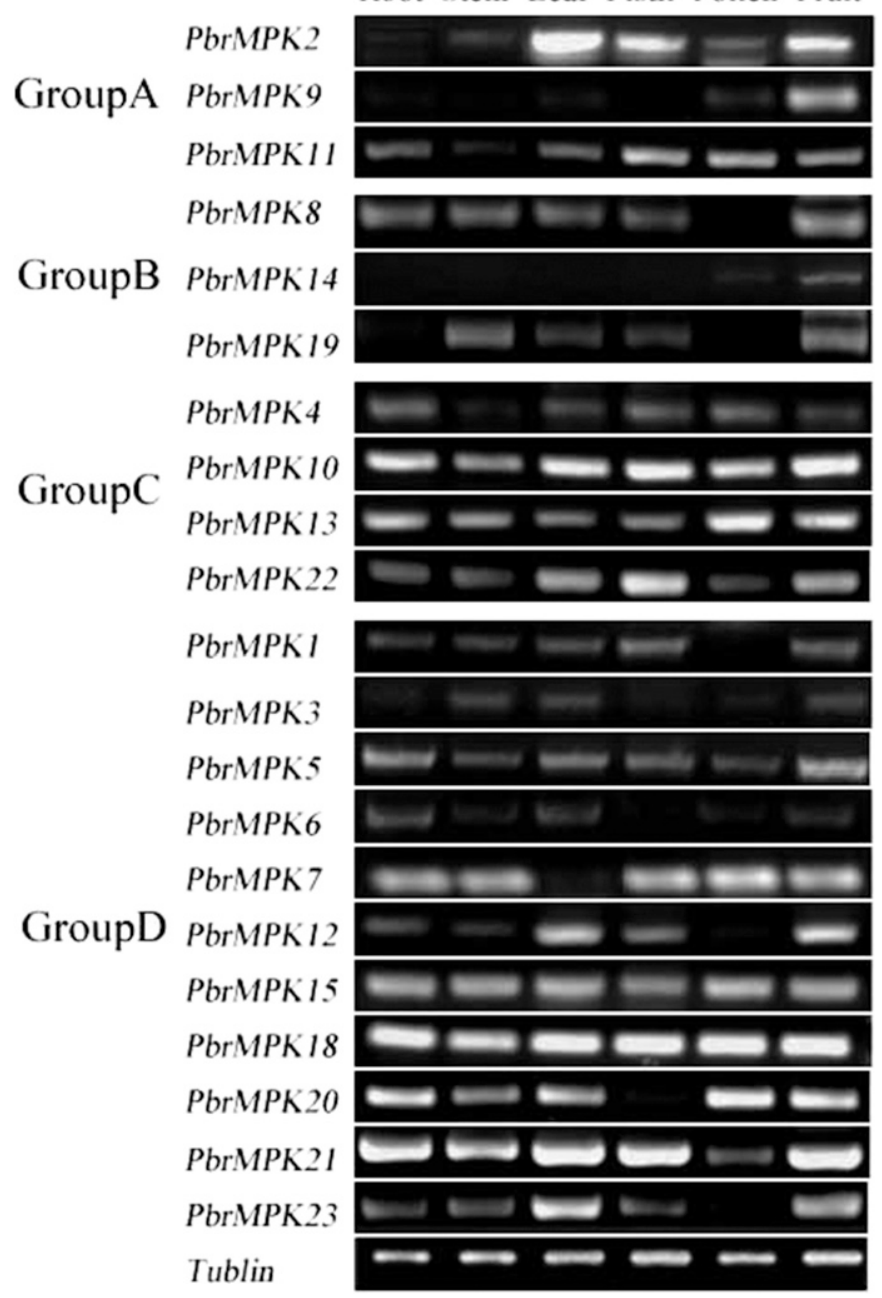

Supplemental Fig. 3. Expression of PbrMAPKs in different tissues of chinese white pear. Reverse transcription polymerase chain reaction (RT-PCR) analysis was performed with root, stem, leaf, seed, pistil, and pollen of chinese white pear. PbrTub-2 gene was used as an internal control. RT-PCR was repeated three times.

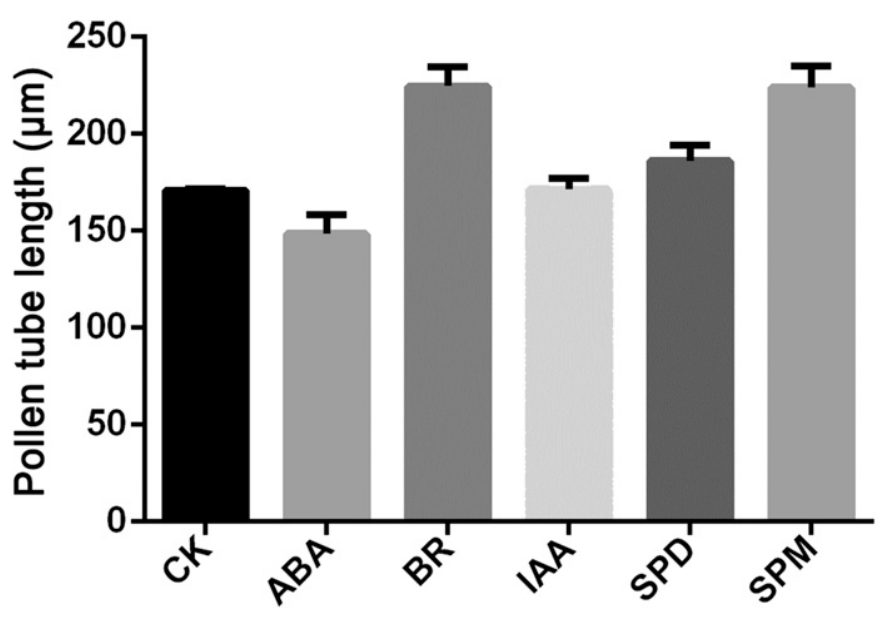

Supplemental Fig. 4. The pollen tube length statistics after five plant hormones treatment. Three replications with 100 pollen grains. The following concentrations: brassinosteroid [BR $\left.\left(0.05 \mu \mathrm{mol} \cdot \mathrm{L}^{-1}\right)\right]$, abscisic acid [ABA $(0.2$ $\left.\mathrm{mmol} \cdot \mathrm{L}^{-1}\right)$ ], indole-3-acetic acid [IAA $\left(0.08 \mathrm{mmol} \cdot \mathrm{L}^{-1}\right)$ ], spermine [SPM $\left.\left(0.005 \mathrm{mmol} \cdot \mathrm{L}^{-1}\right)\right]$, spermidine [SPD $\left.\left(0.05 \mathrm{mmol} \cdot \mathrm{L}^{-1}\right)\right]$, and dimethyl sulfoxide (DMSO) as control. Experiments were repeated three times. Error bar indicates SE. 
Supplemental Table 1. PbrMAPK gene expressions trend under temperature stresses [hot $\left(37^{\circ} \mathrm{C}\right)$, cold $\left(17{ }^{\circ} \mathrm{C}\right)$ ] and various hormones [brassinosteroid (BR), abscisic acid (ABA), indole-3-acetic acid (IAA), spermine (SPM) and spermidine (SPD)] in chinese white pear pollen.

\begin{tabular}{|c|c|c|c|c|c|c|c|}
\hline Gene name & $\mathrm{Hot}^{\mathrm{z}}$ & Cold & $\mathrm{BR}$ & $\mathrm{ABA}$ & SPD & SPM & IAA \\
\hline$\overline{P b r M A P K 1}$ & $\mathrm{D}$ & $\mathrm{U}$ & $\mathrm{U}$ & $\mathrm{U}$ & $\mathrm{U}$ & $\mathrm{U}$ & $\overline{\mathrm{D}}$ \\
\hline PbrMAPK2 & $\mathrm{U}$ & $\mathrm{D}$ & $\mathrm{U}$ & $\mathrm{U}$ & $\mathrm{U}$ & $\mathrm{U}$ & $\mathrm{U}$ \\
\hline PbrMAPK 3 & $\mathrm{D}$ & $\mathrm{D}$ & $\mathrm{D}$ & $\mathrm{D}$ & $\mathrm{D}$ & $\mathrm{U}$ & $\mathrm{D}$ \\
\hline PbrMAPK5 & $\mathrm{U}$ & $\mathrm{D}$ & $\mathrm{D}$ & U & U & $\mathrm{U}$ & $\mathrm{D}$ \\
\hline PbrMAPK6 & $\mathrm{U}$ & $\mathrm{D}$ & $\mathrm{D}$ & $\mathrm{U}$ & $\mathrm{U}$ & $\mathrm{U}$ & $\mathrm{U}$ \\
\hline PbrMAPK7 & $\mathrm{U}$ & $\mathrm{D}$ & $\mathrm{U}$ & $\mathrm{U}$ & $\mathrm{U}$ & $\mathrm{D}$ & U \\
\hline PbrMAPK10 & $\mathrm{U}$ & $\mathrm{D}$ & $\mathrm{U}$ & $\mathrm{D}$ & $\mathrm{U}$ & $\mathrm{U}$ & $\mathrm{U}$ \\
\hline PbrMAPK11 & $\mathrm{U}$ & $\mathrm{D}$ & $\mathrm{U}$ & $\mathrm{U}$ & $\mathrm{U}$ & $\mathrm{U}$ & U \\
\hline PbrMAPK12 & $\mathrm{U}$ & $\mathrm{D}$ & $\mathrm{D}$ & $\mathrm{U}$ & $\mathrm{U}$ & $\mathrm{D}$ & $\mathrm{U}$ \\
\hline PbrMAPK13 & $\mathrm{U}$ & $\mathrm{U}$ & $\mathrm{U}$ & U & $\mathrm{U}$ & $\mathrm{U}$ & U \\
\hline PbrMAPK14 & $\mathrm{U}$ & $\mathrm{U}$ & $\mathrm{D}$ & $\mathrm{U}$ & $\mathrm{U}$ & $\mathrm{U}$ & $\mathrm{D}$ \\
\hline PbrMAPK21 & $\mathrm{U}$ & $\mathrm{D}$ & $\mathrm{U}$ & $\mathrm{D}$ & $\mathrm{D}$ & $\mathrm{U}$ & U \\
\hline PbrMAPK22 & $\mathrm{U}$ & $\mathrm{D}$ & $\mathrm{U}$ & $\mathrm{U}$ & $\mathrm{U}$ & $\mathrm{U}$ & $\mathrm{U}$ \\
\hline PbrMAPK23 & $\mathrm{U}$ & $\mathrm{D}$ & $\mathrm{U}$ & $\mathrm{U}$ & $\mathrm{U}$ & $\mathrm{D}$ & $\mathrm{U}$ \\
\hline
\end{tabular}

${ }^{\mathrm{z}} \mathrm{D}=$ relative expression indicates a downtrend; $\mathrm{U}=$ relative expression indicates a uptrend. 
Supplemental Table 2. The Ka value (nonsynonymous substitutions per site)/Ks value (synonymous substitutions per site) of coding sequences in group A estimated by KaKs Calculator 2.0 (Wang et al., 2012b).

\begin{tabular}{|c|c|c|c|c|c|c|}
\hline Sequence & Method $^{\mathrm{z}}$ & $\mathrm{Ka}$ & $\mathrm{Ks}$ & $\mathrm{Ka} / \mathrm{Ks}$ & Fisher's $P$ & Length (bp) \\
\hline PmuMAPK12-PpeMAPK3 & NG & 0.00232107 & 0.0246943 & 0.0939918 & 0.00213789 & 1110 \\
\hline PmuMAPK2-PpeMAPK6 & NG & 0.00213114 & 0.0401751 & 0.0530462 & 4.09E-06 & 1221 \\
\hline PmuMAPK10-PpeMAPK5 & NG & 0.00349427 & 0.0443467 & 0.0787945 & $1.41 \mathrm{E}-05$ & 1116 \\
\hline PbrMAPK16-PbrMAPK21 & NG & 0.0163772 & 0.114247 & 0.14335 & $2.20 \mathrm{E}-09$ & 1119 \\
\hline PbrMAPK17-PbrMAPK21 & NG & 0.0163772 & 0.114247 & 0.14335 & $2.20 \mathrm{E}-09$ & 1119 \\
\hline PbrMAPK1-PbrMAPK21 & NG & 0.016378 & 0.128064 & 0.127889 & $6.61 \mathrm{E}-11$ & 1119 \\
\hline PbrMAPK7-PbrMAPK15 & NG & 0.0230308 & 0.172188 & 0.133753 & $8.04 \mathrm{E}-14$ & 1110 \\
\hline PbrMAPK3-PbrMAPK23 & NG & 0.0158244 & 0.177016 & 0.0893949 & $2.90 \mathrm{E}-17$ & 1119 \\
\hline PbrMAPK6-PbrMAPK12 & NG & 0.0237246 & 0.189722 & 0.125049 & $9.40 \mathrm{E}-15$ & 1101 \\
\hline PbrMAPK3-PmuMAPK5 & NG & 0.0316693 & 0.262553 & 0.12062 & $3.88 \mathrm{E}-20$ & 1128 \\
\hline PbrMAPK3-PpeMAPK1 & NG & 0.0299092 & 0.275184 & 0.108688 & $1.49 \mathrm{E}-22$ & 1128 \\
\hline PbrMAPK23-PmuMAPK5 & NG & 0.0248524 & 0.283801 & 0.0875698 & $1.57 \mathrm{E}-24$ & 1113 \\
\hline PbrMAPK3-PpeMAPK2 & NG & 0.0582294 & 0.303173 & 0.192067 & $1.21 \mathrm{E}-15$ & 1011 \\
\hline PbrMAPK23-PpeMAPK1 & NG & 0.0200782 & 0.30568 & 0.0656837 & $5.18 \mathrm{E}-29$ & 1113 \\
\hline PbrMAPK5-PpeMAPK6 & NG & 0.0368039 & 0.310433 & 0.118557 & $1.30 \mathrm{E}-25$ & 1218 \\
\hline PbrMAPK5-PmuMAPK2 & NG & 0.0356827 & 0.310487 & 0.114925 & 4.97E-26 & 1218 \\
\hline PbrMAPK7-PpeMAPK4 & NG & 0.0266297 & 0.31621 & 0.0842152 & $1.93 \mathrm{E}-27$ & 1110 \\
\hline PbrMAPK12-PmuMAPK12 & NG & 0.0309873 & 0.321957 & 0.0962467 & $2.29 \mathrm{E}-25$ & 1101 \\
\hline PbrMAPK12-PpeMAPK3 & NG & 0.0309824 & 0.322178 & 0.0961653 & $2.21 \mathrm{E}-25$ & 1101 \\
\hline PbrMAPK21-PpeMAPK10 & NG & 0.0289019 & 0.326703 & 0.0884652 & $6.45 \mathrm{E}-28$ & 1119 \\
\hline PbrMAPK16-PpeMAPK10 & NG & 0.0229052 & 0.327131 & 0.0700184 & $1.38 \mathrm{E}-30$ & 1119 \\
\hline PbrMAPK17-PpeMAPK10 & NG & 0.0229052 & 0.327131 & 0.0700184 & $1.38 \mathrm{E}-30$ & 1119 \\
\hline PbrMAPK21-PmuMAPK6 & NG & 0.0276777 & 0.33393 & 0.0828847 & $4.06 \mathrm{E}-29$ & 1119 \\
\hline FveMAPK1-PpeMAPK8 & NG & 0.0410571 & 0.371037 & 0.110655 & 8.19E-27 & 1104 \\
\hline FveMAPK1-PmuMAPK3 & NG & 0.0398229 & 0.377656 & 0.105448 & 8.01E-28 & 1104 \\
\hline PbrMAPK1-PmuMAPK6 & NG & 0.0216969 & 0.378637 & 0.0573026 & $1.73 \mathrm{E}-36$ & 1119 \\
\hline FveMAPK7-PmuMAPK5 & NG & 0.0413082 & 0.43581 & 0.094785 & 4.10E-33 & 1128 \\
\hline FveMAPK10-PpeMAPK4 & NG & 0.0326596 & 0.436014 & 0.0749049 & $2.21 \mathrm{E}-36$ & 1110 \\
\hline FveMAPK7-PpeMAPK1 & NG & 0.0377212 & 0.440471 & 0.0856383 & $5.59 \mathrm{E}-35$ & 1128 \\
\hline FveMAPK7-PpeMAPK2 & NG & 0.069124 & 0.462661 & 0.149405 & $1.24 \mathrm{E}-24$ & 1011 \\
\hline FveMAPK10-PmuMAPK11 & NG & 0.0326462 & 0.466137 & 0.0700356 & 4.29E-39 & 1110 \\
\hline FveMAPK9-PmuMAPK12 & NG & 0.0688986 & 0.47787 & 0.144179 & $6.13 \mathrm{E}-28$ & 1110 \\
\hline FveMAPK9-PpeMAPK3 & NG & 0.0688874 & 0.478234 & 0.144045 & $5.83 \mathrm{E}-28$ & 1110 \\
\hline FveMAPK10-PbrMAPK7 & NG & 0.0328918 & 0.492237 & 0.0668211 & $1.33 \mathrm{E}-41$ & 1110 \\
\hline FveMAPK9-PbrMAPK6 & NG & 0.0782012 & 0.49461 & 0.158107 & $4.40 \mathrm{E}-27$ & 1122 \\
\hline FveMAPK9-PbrMAPK12 & NG & 0.0649711 & 0.504825 & 0.1287 & $2.82 \mathrm{E}-30$ & 1101 \\
\hline PbrMAPK20-PmuMAPK10 & NG & 0.0266011 & 0.510856 & 0.0520715 & $1.08 \mathrm{E}-46$ & 1116 \\
\hline FveMAPK7-PbrMAPK3 & NG & 0.0502181 & 0.516275 & 0.09727 & $6.18 \mathrm{E}-38$ & 1155 \\
\hline PbrMAPK20-PpeMAPK5 & NG & 0.0253974 & 0.526631 & 0.0482261 & $8.96 \mathrm{E}-49$ & 1116 \\
\hline FveMAPK2-PbrMAPK18 & NG & 0.0444694 & 0.539888 & 0.0823678 & $1.28 \mathrm{E}-42$ & 1158 \\
\hline FveMAPK2-PpeMAPK6 & NG & 0.0402138 & 0.547568 & 0.0734407 & $2.31 \mathrm{E}-45$ & 1173 \\
\hline FveMAPK2-PmuMAPK2 & NG & 0.0413766 & 0.547687 & 0.0755479 & $7.46 \mathrm{E}-45$ & 1173 \\
\hline FveMAPK10-PbrMAPK15 & NG & 0.0338844 & 0.551249 & 0.0614685 & $5.63 \mathrm{E}-46$ & 1110 \\
\hline FveMAPK2-PbrMAPK5 & NG & 0.0443366 & 0.55696 & 0.0796045 & $7.31 \mathrm{E}-44$ & 1173 \\
\hline
\end{tabular}


Supplemental Table 2. Continued.

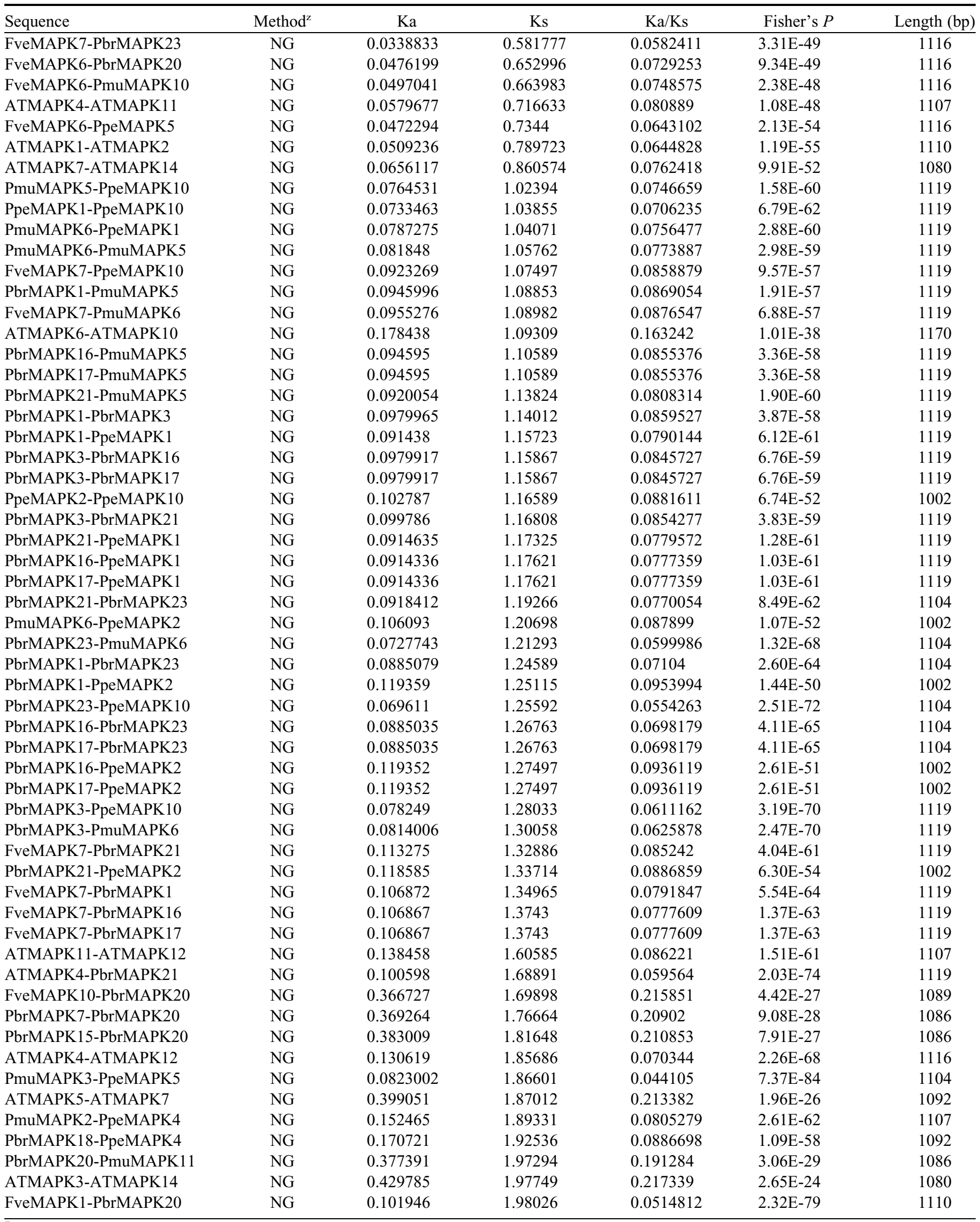

${ }^{\mathrm{z}} \mathrm{NG}=$ nonuniform grid. 